Energy Technology Division

Encrgy Technology Division

Energy Technology Division

Energy Technology Division

Energy Technology Division

Energy Technology Division

Encrey Technology Division

Energy Technology Division

Energy Technology Division

Energy Technology Division

Energy Technology Division

Energy Technology Division

Energy Technology Division

Energy Technology Division

Energy Technology Division

Energy Technology Division

Energy Technology Division

Energy Technology Division

Energy Technology Division

Energy Technology Division

Energy Technology Division

Energy Technology Division

Energy Technology Division

Energy Technology Division

Encrgy Technology Division

Energy Technology Division

Energy Technology Division

Energy Technology Division

Energy Technology Division

Energy Technology Division

Energy Technology Division

Energy Technology Division

Energy Technology Division

\title{
Nonlinear Dynamics of a Stack/Cable System
}

\author{
by Y. Cai and S. S. Chen
}

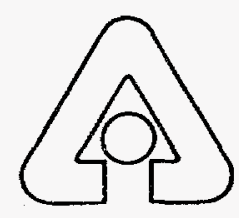

Argonne National Laboratory, Argonne, Illinois 60439

operated by The University of Chicago

for the United States Department of Energy under Contract W-31-109-Eng-38

\section{Energy Technology Division \\ Energy Technology Division \\ Energy Technology Division \\ Energy Technology Division \\ Energy Technology Division \\ Energy Technology Divislon \\ Energy Technology Division \\ Energy Technology Division}


Argonne National Laboratory, with facilities in the states of Illinois and Idaho, is owned by the United States government, and operated by The University of Chicago under the provisions of a contract with the Department of Energy.

\section{DISCLAIMER}

This report was prepared as an account of work sponsored by an agency of the United States Government. Neither the United States Government nor any agency thereof, nor any of their employees, makes any warranty, express or implied, or assumes any legal liability or responsibility for the accuracy, completeness, or usefulness of any information, apparatus, product, or process disclosed, or represents that its use would not infringe privately owned rights. Reference herein to any specific commercial product, process, or service by trade name, trademark, manufacturer, or otherwise, does not necessarily constitute or imply its endorsement, recommendation, or favoring by the United States Government or any agency thereof. The views and opinions of authors expressed herein do not necessarily state or reflect those of the United States Government or any agency thereof.

Reproduced from the best available copy.

Available to DOE and DOE contractors from the Office of Scientific and Technical Information P.O. Box 62

Oak Ridge, TN 37831

Prices available from (615) 576-8401

Available to the public from the National Technical Information Service

U.S. Department of Commerce 5285 Port Royal Road Springfield, VA 22161 


\section{DISCLAIMER}

Portions of this document may be illegible in electronic image products. Images are produced from the best available original document. 


\section{Nonlinear Dynamics of a Stack/Cable System}

by

Y. Cai and S. S. Chen

Energy Technology Division

July 1995

Work supported by

U.S. DEPARTMENT OF ENERGY

Office of Basic Energy Sciences

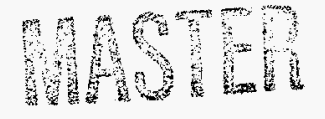





\section{Contents}

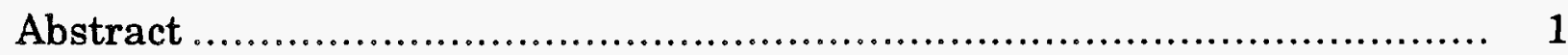

1 Introduction ..................................................................... 1

1.1 Background ................................................................ 2

1.2 Observations of Stack/Wire Oscillations ................................... 3

1.3 Scoping Calculations and Predictions ..................................... 5

1.4 Identification of Vibration Mechanisms................................... 7

2 Vortex-Shedding-Induced Stack Lock-In Vibrations............................ 9

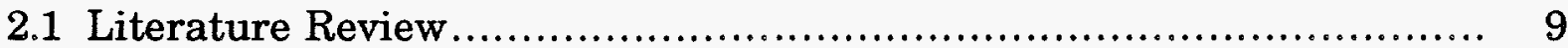

2.2 Unsteady-Flow Theory .............................................. 11

2.3 Equation of Motion for Vortex-Induced Stack Vibration................... 13

2.4 Lock-In Resonance of Stack ............................................. 15

3 Parametric and External Resonances of Nonlinear Cables.................... 16

3.1 Literature Review......................................................... 16

3.2 Nonlinear System of Elastic Cables........................................ 17

3.3 Equation of Motion for an Elastic Cable .................................. 18

3.4 Parametric Resonance of Cable........................................ 23

3.5 Numerical Simulation for Parametric and External Resonances....... 27

4 Dynamics of a Stack/Cable System.................................................. 33

4.1 Coupled Model of a Stack/Cable System ................................... 33

4.2 Dynamic Response of a Stack/Cable System ............................. 34

5 Techniques to Suppress Excessive Vibration...................................... 39

5.1 Short-Term Solution ........................................................ 39

5.2 Long-Term Solution................................................... 40

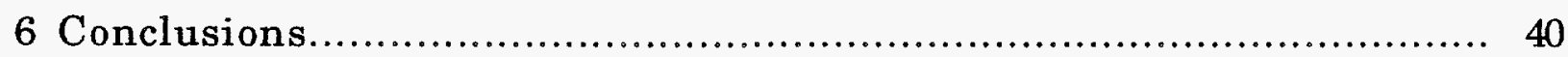

Acknowledgments................................................................. 42

References........................................................................... 42

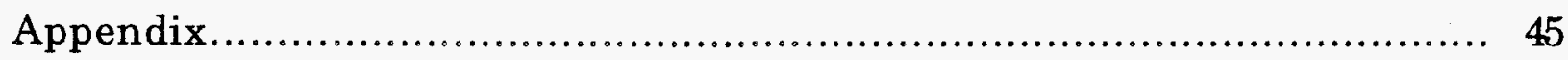




\section{Figures}

1 Stack supported by guy wires at four levels ............................. 2

2 Strouhal number as a function of Reynolds number ................... 4

3 Frequencies and mode shapes of first four natural modes of stack..... 4

4 Circular cylinder oscillating in crossflow............................. 11

5 Fluid-stiffness and fluid-damping coefficients for a single cylinder,

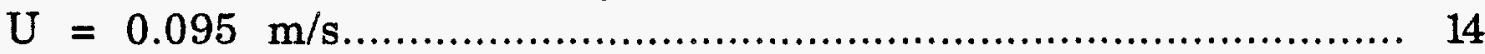

$6 \quad$ Vortex-shedding frequencies as a function of wind speed .............. 15

$\begin{array}{ll}7 & \text { RMS values of stack motion as a function of wind speed ................ } 16\end{array}$

8 Cable supported in angled configuration ............................. 19

9 Unstable region of principal parametric resonance .................. 26

10 RMS displacements of cable at $x=0.500$ and $x=0.125$ versus excitation frequency ratio $f_{r}$ with various excitation amplitudes $w_{0} \ldots 28$

11 Maximum cable responses with various excitation amplitudes $\mathrm{w}_{0} \ldots .28$

12 Power spectral densities of cable displacement at $\mathrm{x}=0.125$ with excitation amplitude $\mathrm{w}_{0}=0.0003 \ldots$

13 RMS displacements of cable at $\mathrm{x}=0.500$ near excitation frequency

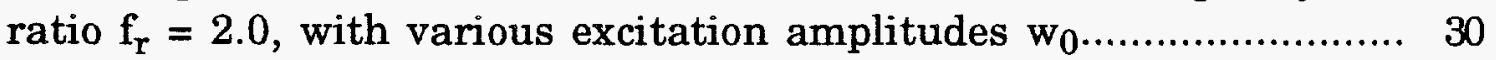

14 Time histories of cable displacements with a fixed frequency ratio

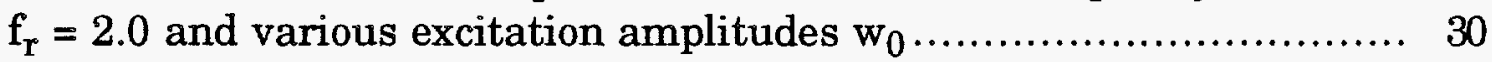

15 Time histories of first two modes with fixed frequency ratio $f_{r}=2.0$ and various excitation amplitudes

16 RMS displacements of cable at $\mathrm{x}=0.500$ near excitation frequency

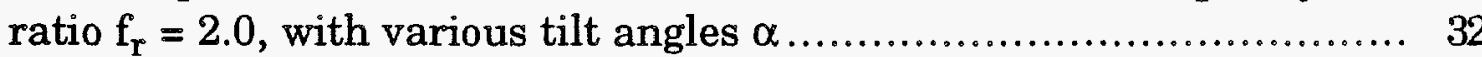

17 RMS displacements of cable at $\mathrm{x}=0.500$ near excitation frequency

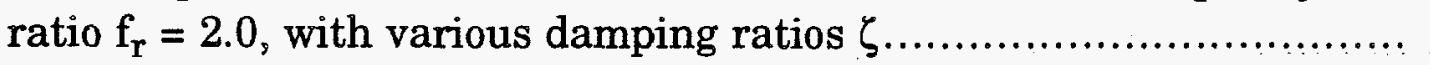


18 Nondimensional RMS displacements of cable at $\mathrm{x} / \ell=0.5$

19 Power spectral densities of cable displacements at $x / \ell=0.5$

20 Nondimensional RMS displacements of cable at $x / \ell=0.5$ with various cable tensions

21 Nondimensional RMS displacements of cable at $\mathrm{x} / \ell=0.5$ with various cable dampings.

22 Nondimensional RMS displacements of cable at $x / \ell=0.5$ with $\mathrm{C}_{\mathrm{L}}^{\prime}=1.0$ 38

\section{Tables}

1 Dimensional parameters and material properties of system shown in Fig. 1

2 Nondimensional parameters of system shown in Fig. 1 at wind speed of $15 \mathrm{~m} / \mathrm{s}$

3 Natural frequencies of first 20 modes of the 10 models, computed with a finite-element code.

4 Fundamental frequencies of guy wires at four different levels as a function of tension for original guy wires

5 Fundamental frequencies of guy wires at four levels as a function of tension for guy wires with cross-sectional area twice that of original guy wires 


\title{
Nonlinear Dynamics of a Stack/Cable System
}

by

Y. Cai and S. S. Chen

\begin{abstract}
In this study, we developed a coupled model of wind-induced vibration of a stack, based on an unsteady-flow theory and nonlinear dynamics of the stack's heavy elastic suspended cables. Numerical analysis was performed to identify excitation mechanisms. The stack was found to be excited by vortex shedding. Once lock-in resonance occurred, the cables were excited by the transverse motion of the stack. Large-amplitude oscillations of the cables were due to parametric resonance. Appropriate techniques have been proposed to alleviate the vibration problem.
\end{abstract}

\section{Introduction}

A stack supported by cable guy wires at four levels was susceptible to largeamplitude oscillations and some of the guy wires at the lower two levels were damaged when wind speed exceeded $15 \mathrm{~m} / \mathrm{s}$ for a period of time. The excitation mechanism was identified through scoping calculations, analytical prediction with a finite-element code, and observation of the stack/wire response (Chen and Cai 1992). The stack was found to be excited by vortex shedding. Once lock-in resonance occurred, the guy wires were parametrically excited at the upper end by the transverse motion of the stack. Large-amplitude oscillations of the guy wires were observed. The lowest natural frequency of the guy wires at the lower two levels was approximately one-half that of the third mode of the stack. This is a typical parametric resonance (Cai and Chen 1994).

We have developed a coupled nonlinear dynamic model of the wind-induced vibration of the stack (based on an unsteady-flow theory) and of the heavy elastic suspended cables whose upper ends are subject to bending vibration of the stack. The response characteristics of the stack and cables under various conditions are presented. Numerical analysis of the coupled system exhibits the effect of fluid/structure interaction and cable parameters on parametric and external resonances of cables. The dominant excitation mechanisms are lock-in resonance of the stack by vortex shedding and parametric resonance of suspended cables by stack motion at their support ends. Appropriate techniques are proposed to alleviate the vibration problem. 


\subsection{Background}

The stack discussed in this report was a cantilevered tube supported by cable guy wires at four levels, as shown in Fig. 1 . At each level, three guy wires were attached, at $120^{\circ}$ intervals. Strakes were attached to the top $30 \mathrm{~m}$ of the stack to prevent vortex-excited oscillations. When the wires were not tensioned by large forces, no significant vibration was noted. When the wires were tensioned to higher values, severe oscillations of the stack/wire system were observed. Some general observations can be described as follows: (1) When wind speed was $<7 \mathrm{~m} / \mathrm{s}$, the stack and wires were stable and no significant vibration was noticed; (2) as the wind speed increased to $\approx 10 \mathrm{~m} / \mathrm{s}$, the stack/wire system vibrated gently; and (3) when the wind speed reached $\approx 15 \mathrm{~m} / \mathrm{s}$, the stack was dancing and the wires were galloping severely.

The dimensional parameters and material properties of the system shown in Fig. 1 (referred to as the original system) are summarized in Table 1; nondimensional parameters are summarized in Table 2. The damping ratio $\zeta$ for the stack and wires is assumed to be a few percent. The Young's modulus of helically woven guy wire is assumed to be $6.2 \times 10^{10}-10.3 \times 10^{10} \mathrm{~N} / \mathrm{m}^{2}$ (Blevins 1979). Figure 2 shows the relationship of Strouhal number to Reynolds number.

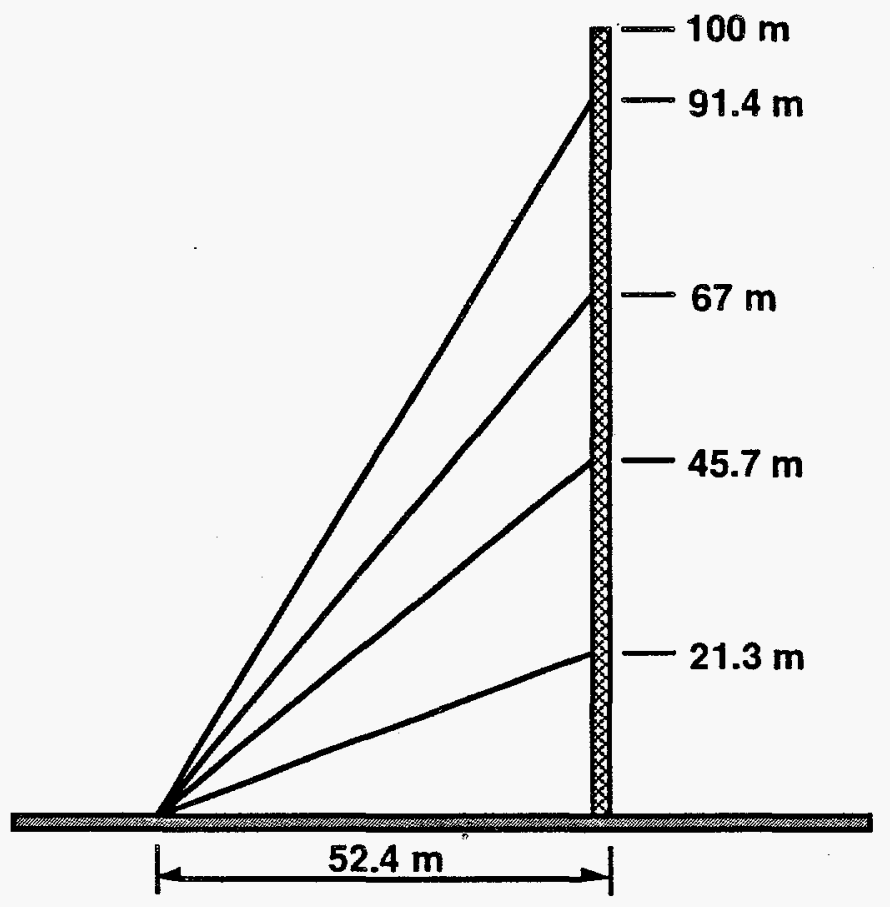

Fig. 1. Stack supported by guy wires at four levels 
Table 1. Dimensional parameters and material properties of system shown in Fig. 1

Stack

Length

Outside diameter $\left(\mathrm{D}_{0}\right)$

$100 \mathrm{~m}$

Inside diameter $\left(\mathrm{D}_{\mathbf{i}}\right)$

$1.238 \mathrm{~m}$

Density $(\rho)$

$1.219 \mathrm{~m}$

Young's modulus (E)

$1.66 \mathrm{~g} / \mathrm{cm}^{3}$

Mass per unit length (m)

$8.09 \times 10^{4} \mathrm{~kg} / \mathrm{cm}^{2}$

$61.08 \mathrm{~kg} / \mathrm{m}$

Guy Wires

Density $\left(\rho_{\mathrm{w}}\right)$

$7.83 \mathrm{~g} / \mathrm{cm}^{3}$

Outside diameter $\left(D_{\mathrm{w}}\right)$

$1.59 \mathrm{~cm}$

Cross-sectional area (A)

$1.54 \mathrm{~cm}^{2}$

Mass per unit length $\left(\mathrm{m}_{\mathrm{w}}\right)$

$1.20 \mathrm{~kg} / \mathrm{m}$

Table 2. Nondimensional parameters of system shown in Fig. 1 at wind speed of $15 \mathrm{~m} / \mathrm{s}$

\begin{tabular}{lll}
\hline Nondimensional Parameter & Stack & Wire \\
\hline Reynolds number (Re) & $1,090,000$ & 14,000 \\
Strouhal number (St) & $0.2-0.4$ & 0.2 \\
Mass-damping parameter & 2 & 243 \\
\hline
\end{tabular}

\subsection{Observations of Stack/Wire Oscillations}

Vibration characteristics were observed and the following important characteristics were noted.

Stack: The oscillation frequency of the stack was $\approx 2.3 \mathrm{~Hz}$; the dominant stack vibration appeared to be transverse to the wind. The upper portion of the stack above the third level of guy wires vibrated very little. The dominant motion of the stack was below the third level of guy wires, in particular, between the lower two levels of guy wires (this corresponds to the third mode given in Fig. 3). 


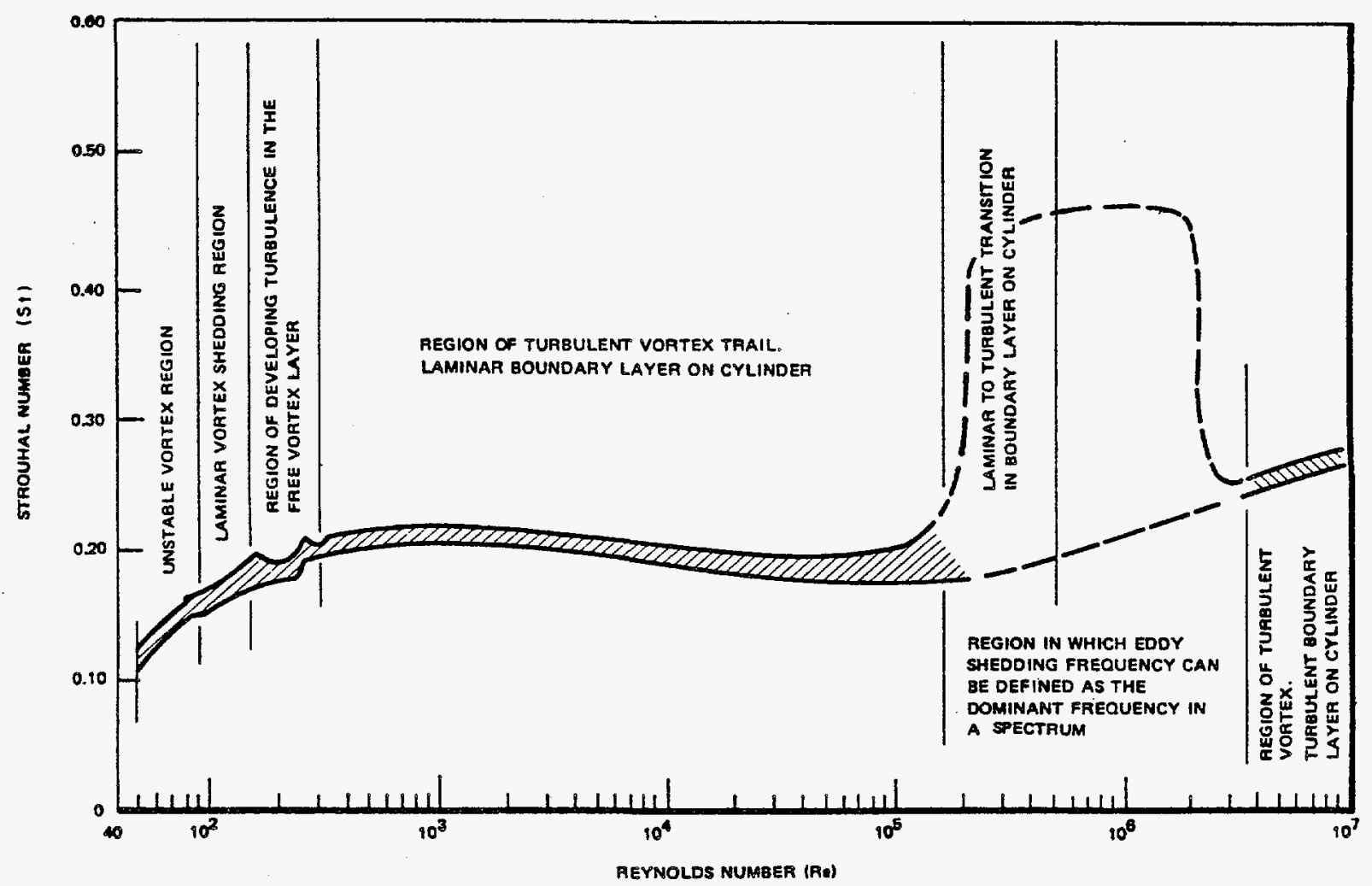

Fig. 2. Strouhal number (St) as a function of Reynolds number
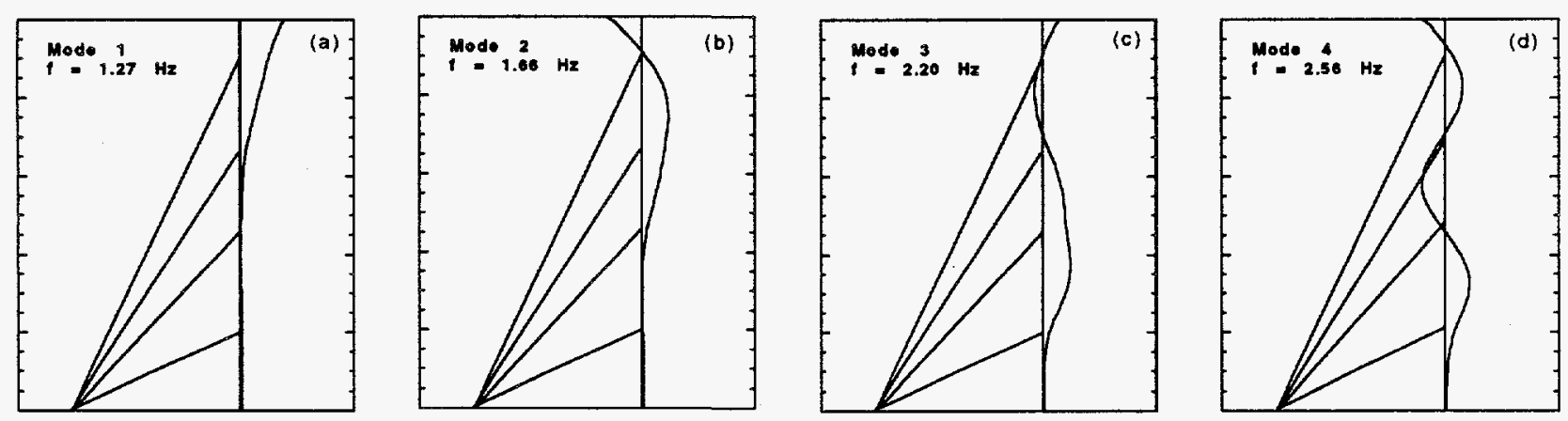

Fig. 3. Frequencies and mode shapes of first four natural modes of stack

Guy Wires: The oscillation frequency of the guy wires at the lower two levels was $\approx 1.2 \mathrm{~Hz}$, and the oscillation mode of the wires appeared to be the first natural mode. Large-amplitude oscillations were associated with the wires at the lower two levels only. 
The most important information from these observations is that the stack oscillation frequency was approximately twice that of the wires, and that the critical mode of the stack was associated with the dominant vibration between the first $(21.3 \mathrm{~m})$ and third $(67.0 \mathrm{~m})$ levels of guy wires, and not with motion above the third level of guy wires.

\subsection{Scoping Calculations and Predictions}

Ten models of the original system were analyzed to simulate the dynamic characteristics of the system and understand the effects of guy wires:

Model 1. Original system (shown in Fig. 1 and described in Table 1): Stack supported by elastic springs (wires modeled as springs.)

Model 2. Guy wires added at $79.2 \mathrm{~m}$.

Model 3. Guy wires added at $100 \mathrm{~m}$.

Model 4. Guy wires at 21.3 and $67.0 \mathrm{~m}$ moved to 22.9 and $68.6 \mathrm{~m}$, respectively; therefore, all four levels of guy wires were equally spaced.

Model 5. Guy wires equally spaced at five levels, i.e., at $19.8,39.6,59.4,79.2$, and $99 \mathrm{~m}$.

Model 6. Original system with thicker guy wires, of cross-sectional area twice that given in Table 1.

Model 7. Original system with thicker guy wires (cross-sectional area twice that given in Table 1) at 21.3 and $45.7 \mathrm{~m}$.

Model 8. Original system with thicker guy wires at 67.0 and $91.4 \mathrm{~m}$, i.e., guy wires with cross-sectional area twice that given in Table 1.

Model 9. Original system with thicker guy wires (cross-sectional area twice that given in Table 1) at 45.7 and $91.4 \mathrm{~m}$.

Model 10. Guy wires added at 56.4 and $79.2 \mathrm{~m}$.

- In all analytical models, guy wires were modeled as springs and the stack as a Bernoulli-Euler beam with the lower end fixed and the top end free. The effect of spoilers on the vibration of the coupled stack/wire system was not considered. The guy wires were helically woven wires and their wire modulus was taken as $\approx 50 \%$ of the modulus of elasticity of the component wires (Blevins 1979). 
The first 20 modes of all models were analyzed with the finite-element code MSC-PAL. The natural frequencies are given in Table 3. The natural frequencies and natural modes of the first model for the first four modes are given in Fig. 3. Although the natural frequencies in the lower modes varied somewhat, in the higher modes, all 10 models exhibited approximately the same natural frequencies. This means that the coupling between the stack and guy wires is important only for low-frequency modes. In the higher frequency range, the motion was basically associated with the motion of the stack.

The natural frequencies of the existing guy wires were also calculated as a function of tension at the four levels. The fundamental frequencies for the four levels of guy wires are given in Table 4. The natural frequencies of higher modes are equal to the multiples of those given in Table 4. The natural frequencies of the guy wires with cross-sectional area twice that of the original guy wires at the four levels were also calculated. The results are given in Table 5.

Table 3. Natural frequencies ( $\mathrm{Hz}$ ) of first 20 modes of the 10 models, computed with a finite-element code

\begin{tabular}{|c|c|c|c|c|c|c|c|c|c|c|}
\hline \multirow[b]{2}{*}{ Modes } & \multicolumn{10}{|c|}{ Model } \\
\hline & 1 & 2 & 3 & 4 & 5 & 6 & 7 & 8 & 9 & 10 \\
\hline 1 & 1.27 & 1.36 & 1.47 & 1.28 & 1.51 & 1.75 & 1.75 & 1.27 & 1.28 & 1.37 \\
\hline 2 & 1.65 & 1.95 & 2.07 & 1.65 & 1.99 & 1.99 & 2.00 & 1.67 & 1.99 & 2.21 \\
\hline 3 & 2.19 & 2.25 & 2.32 & 2.23 & 2.53 & 2.74 & 2.21 & 2.62 & 2.26 & 2.31 \\
\hline 4 & 2.55 & 2.72 & 2.90 & 2.60 & 3.03 & 2.78 & 2.64 & 2.77 & 2.74 & 3.21 \\
\hline 5 & 3.99 & 4.00 & 4.01 & 3.72 & 4.14 & 4.74 & 4.02 & 4.74 & 4.71 & 4.00 \\
\hline 6 & 4.74 & 4.75 & 4.87 & 4.76 & 4.74 & 5.13 & 4.94 & 4.94 & 4.92 & 4.79 \\
\hline 7 & 6.36 & 6.36 & 6.44 & 6.48 & 6.51 & 6.68 & 6.39 & 6.67 & 6.47 & 6.48 \\
\hline 8 & 8.41 & 8.45 & 8.48 & 8.45 & 8.48 & 8.71 & 8.48 & 8.64 & 8.67 & 8.45 \\
\hline 9 & 10.75 & 10.78 & 10.79 & 10.71 & 10.71 & 11.16 & 10.83 & 11.10 & 11.04 & 10.86 \\
\hline 10 & 12.99 & 13.01 & 13.03 & 12.95 & 13.24 & 13.11 & 13.02 & 13.08 & 13.08 & 13.02 \\
\hline 11 & 15.75 & 15.75 & 15.78 & 15.77 & 15.71 & 15.86 & 15.78 & 15.83 & 15.77 & 15.80 \\
\hline 12 & 18.76 & 18.76 & 18.79 & 18.83 & 18.79 & 18.85 & 18.81 & 18.81 & 18.84 & 18.79 \\
\hline 13 & 22.09 & 22.10 & 22.11 & 22.12 & 22.09 & 22.24 & 22.10 & 22.23 & 22.19 & 22.12 \\
\hline 14 & 25.53 & 25.54 & 25.55 & 25.48 & 25.60 & 25.61 & 25.54 & 25.61 & 25.61 & 25.58 \\
\hline 15 & 29.27 & 29.27 & 29.28 & 29.26 & 29.36 & 29.34 & 29.29 & 29.32 & 29.30 & 29.27 \\
\hline 16 & 33.21 & 33.21 & 33.23 & 33.27 & 33.22 & 33.23 & 33.22 & 33.22 & 33.22 & 33.25 \\
\hline 17 & 37.51 & 37.51 & 37.52 & 37.55 & 37.51 & 37.57 & 37.52 & 37.57 & 37.55 & 37.52 \\
\hline 18 & 42.04 & 42.05 & 42.05 & 42.03 & 42.05 & 42.12 & 42.06 & 42.11 & 42.11 & 42.07 \\
\hline 19 & 46.78 & 46.78 & 46.79 & 46.76 & 46.82 & 46.82 & 46.78 & 46.81 & 46.81 & 46.80 \\
\hline 20 & 51.81 & 51.82 & 51.82 & 51.82 & 51.88 & 51.84 & 51.82 & 51.83 & 51.82 & 51.82 \\
\hline
\end{tabular}


Table 4. Fundamental frequencies of guy wires $(\mathrm{Hz})$ at four different levels as a function of tension for original guy wires

\begin{tabular}{rcccc}
\hline & \multicolumn{3}{c}{ Level } \\
Tension & 1 & 2 & 3 & 4 \\
\cline { 2 - 5 }$(\mathrm{kg})$ & $(21.3 \mathrm{~m})$ & $(45.7 \mathrm{~m})$ & $(67.0 \mathrm{~m})$ & $(91.4 \mathrm{~m})$ \\
\hline 500 & 0.566 & 0.459 & 0.375 & 0.303 \\
1000 & 0.800 & 0.650 & 0.531 & 0.429 \\
1500 & 0.980 & 0.796 & 0.650 & 0.525 \\
2000 & 1.131 & 0.919 & 0.751 & 0.606 \\
2500 & 1.265 & 1.027 & 0.840 & 0.678 \\
3000 & 1.386 & 1.125 & 0.920 & 0.743 \\
3500 & 1.497 & 1.216 & 0.993 & 0.802 \\
4000 & 1.600 & 1.300 & 1.062 & 0.857 \\
4500 & 1.697 & 1.378 & 1.126 & 0.909 \\
5000 & 1.789 & 1.453 & 1.187 & 0.959 \\
5500 & 1.876 & 1.524 & 1.245 & 1.005 \\
6000 & 1.960 & 1.592 & 1.301 & 1.050 \\
6500 & 2.040 & 1.657 & 1.354 & 1.093 \\
7000 & 2.117 & 1.719 & 1.405 & 1.134 \\
7500 & 2.191 & 1.779 & 1.454 & 1.174 \\
8000 & 2.263 & 1.838 & 1.502 & 1.213 \\
8500 & 2.332 & 1.894 & 1.548 & 1.250 \\
9000 & 2.400 & 1.949 & 1.593 & 1.286 \\
9500 & 2.466 & 2.003 & 1.637 & 1.321 \\
10000 & 2.530 & 2.055 & 1.679 & 1.356 \\
\hline
\end{tabular}

The analytical results from these ten models are useful for determining the cause of large-amplitude oscillations and recommending proper courses of action for the resolution of excessive vibration.

\subsection{Identification of Vibration Mechanisms}

On the basis of scoping calculations, analytical results, and observation, the excitation mechanisms were identified as follows:

Stack: $\quad$ Lock-in resonance by vortex shedding

Guy Wires: Parametric resonance excited by stack oscillations

These two mechanisms are supported by the following characteristics: 
Table 5. Fundamental frequencies of guy wires $(\mathrm{Hz})$ at four levels as a function of tension for guy wires with cross-sectional area twice that of original guy wires

\begin{tabular}{|c|c|c|c|c|}
\hline \multirow[b]{2}{*}{$\begin{array}{c}\text { Tension } \\
(\mathrm{kg})\end{array}$} & \multicolumn{4}{|c|}{ Level } \\
\hline & $\begin{array}{c}1 \\
(21.3 \mathrm{~m})\end{array}$ & $\begin{array}{c}2 \\
(45.7 \mathrm{~m})\end{array}$ & $\begin{array}{c}3 \\
(67.0 \mathrm{~m})\end{array}$ & $\begin{array}{c}4 \\
(91.4 \mathrm{~m})\end{array}$ \\
\hline 500 & 0.400 & 0.325 & 0.265 & 0.214 \\
\hline 1000 & 0.566 & 0.459 & 0.375 & 0.303 \\
\hline 1500 & 0.693 & 0.563 & 0.460 & 0.371 \\
\hline 2000 & 0.800 & 0.650 & 0.531 & 0.429 \\
\hline 2500 & 0.894 & 0.726 & 0.594 & 0.479 \\
\hline 3000 & 0.980 & 0.796 & 0.650 & 0.525 \\
\hline 3500 & 1.058 & 0.860 & 0.702 & 0.567 \\
\hline 4000 & 1.131 & 0.919 & 0.751 & 0.606 \\
\hline 4500 & 1.200 & 0.975 & 0.796 & 0.643 \\
\hline 5000 & 1.265 & 1.027 & 0.840 & 0.678 \\
\hline 5500 & 1.327 & 1.078 & 0.880 & 0.711 \\
\hline 6000 & 1.386 & 1.125 & 0.920 & 0.743 \\
\hline 6500 & 1.442 & 1.171 & 0.957 & 0.773 \\
\hline 7000 & 1.497 & 1.216 & 0.993 & 0.802 \\
\hline 7500 & 1.549 & 1.258 & 1.028 & 0.830 \\
\hline 8000 & 1.600 & 1.300 & 1.062 & 0.857 \\
\hline 8500 & 1.649 & 1.340 & 1.095 & 0.884 \\
\hline 9000 & 1.697 & 1.378 & 1.126 & 0.909 \\
\hline 9500 & 1.744 & 1.416 & 1.157 & 0.934 \\
\hline 10000 & 1.789 & 1.453 & 1.187 & 0.959 \\
\hline
\end{tabular}

Stack

- At $15 \mathrm{~m} / \mathrm{s}$, the vortex-shedding frequency is $\approx 2.2$ to $4.4 \mathrm{~Hz}$, which is close to the five natural frequencies of the second to sixth modes of Model 1. This is one of the requirements for lock-in resonance.

- The mass-damping parameter is 2 , which is $<32$. This is the second requirement for lock-in resonance.

- The observed vibration mode was approximately the same as the mode shape of the third mode of Model 1, which is shown in Fig. 3. Because the upper portion of the stack contained spoilers and the lower portion did not, this particular mode was vulnerable to 
vortex-shedding-induced resonance because of a large participation factor associated with vortex shedding.

Specifically, we concluded that the stack vibration was excited by vortex shedding at the lower portion, below the third level of guy wires associated with the third mode of Model 1.

\section{Guy Wires}

- The vortex-shedding frequency was much larger than the natural frequencies of the guy wires and the mass-damping parameter was much larger than 32. Therefore, vortex-induced resonance was not possible.

- The wires may have been excited by turbulence. However, turbulence-induced vibration of guy wires is usually small. The large-amplitude galloping of the guy wires could not have been induced by turbulence.

- The lowest natural frequency of the guy wires at the lower two levels was approximately one-half that of the third mode of the stack (Model 1). This made parametric resonance of the guy wires possible. The guy wires were parametrically excited by transverse vibration of the stack, i.e., the guy wires were excited at the upper end by the bending motion of the stack.

It may be firmly concluded that the guy wires at the lower two levels were excited by the transverse motion of the stack. The wire oscillation frequency was one-half that of the stack oscillations. This is a typical parametric resonance.

\section{Vortex-Shedding-Induced Stack Lock-In Vibrations}

\subsection{Literature Review}

Vortex shedding across a bluff body has been studied for more than 100 years. Many reviews on this subject are available (Bearman 1984; Griffin 1982; King 1977; Morkovin 1964; Sarpkaya 1979; Sarpkaya and Isaacson 1981). Because of its engineering significance and academic interest, the phenomenon of structural response to vortex shedding has received wide attention. 
When the natural frequency of a structure is close to the vortex-shedding frequency, lock-in resonance may occur, depending on the mass-damping parameter. Many mathematical models have been developed to characterize the response in the lock-in region. These models can be divided into two groups:

- Single-Degree-of-Freedom Models. Fluid excitation forces and their effects are included in the equation of a single-degree-of-freedom system. The fluid effects are characterized in terms of fluid damping, fluid stiffness, or other fluid-force coefficients that are a function of system parameters and may be linear or nonlinear (Sarpkaya 1979; Scanlan 1977; Simiu and Scanlan 1986; Staubli 1983; Vickery and Basu 1983).

- Coupled Models. The structural system and fluid flow are modeled as two systems coupled by the vortex-shedding process. Both structure and fluid are modeled as a single-degree-of-freedom system. The coupling between the two systems includes different forms (Birkhoff 1953; Blevins 1977; Dowell 1981; Hartlen and Currie 1970; Skop and Griffin 1973).

It is fair to say that not all aspects of the complex interaction process of structural motion and flow field are included in the models. In addition, the exact mechanism of the lock-in resonance is still not well quantified.

Another approach, which uses direct numerical simulation, has also been taken to predict structure response without looking into detailed mechanisms (Ikegawa et al. 1993; Sadaoka and Umegaki 1993). Some time will be required to develop a reliable numerical method for the prediction of structural response with confidence.

Chen et al. (1995) presented an unsteady-flow theory for structural response to vortex shedding. The theory may be considered a single-degree-of-freedom model. The flow effects are characterized as fluid damping and fluid stiffness, which are, in turn, functions of other system parameters such as oscillation amplitude, reduced flow velocity, and Reynolds number. Once fluid damping and fluid stiffness are measured experimentally, the model can be used to predict structural response. From the unsteady-flow model, some general characteristics can also be identified.

The unsteady-flow theory was employed in this study to predict vortexshedding-induced stack vibration. 


\subsection{Unsteady-Flow Theory}

Consider a circular cylinder oscillating in crossflow, as shown in Figure 4. The fluid is flowing at velocity $U$. The displacement components of the cylinder in the $\mathrm{x}$ and $\mathrm{y}$ directions are $\mathrm{u}$ and $\mathrm{v}$, respectively. The motion-dependent fluid-force components that act on the cylinder in the $x$ and $y$ directions are $f$ and $g$, respectively, and are given (Chen 1987) as

$$
\begin{aligned}
& f=-\rho \pi R^{2}\left(\alpha \frac{\partial^{2} u}{\partial t^{2}}+\sigma \frac{\partial^{2} v}{\partial t^{2}}\right)+\frac{\rho U^{2}}{\omega}\left(\alpha^{\prime} \frac{\partial u}{\partial t}+\sigma^{\prime} \frac{\partial v}{\partial t}\right)+\rho U^{2}\left(\alpha^{\prime \prime} u+\sigma^{\prime \prime} v\right) \\
& g=-\rho \pi R^{2}\left(\tau \frac{\partial^{2} u}{\partial t^{2}}+\beta \frac{\partial^{2} v}{\partial t^{2}}\right)+\frac{\rho U^{2}}{\omega}\left(\tau^{\prime} \frac{\partial u}{\partial t}+\beta^{\prime} \frac{\partial v}{\partial t}\right)+\rho U^{2}\left(\tau^{\prime \prime} u+\beta^{\prime \prime} v\right)
\end{aligned}
$$
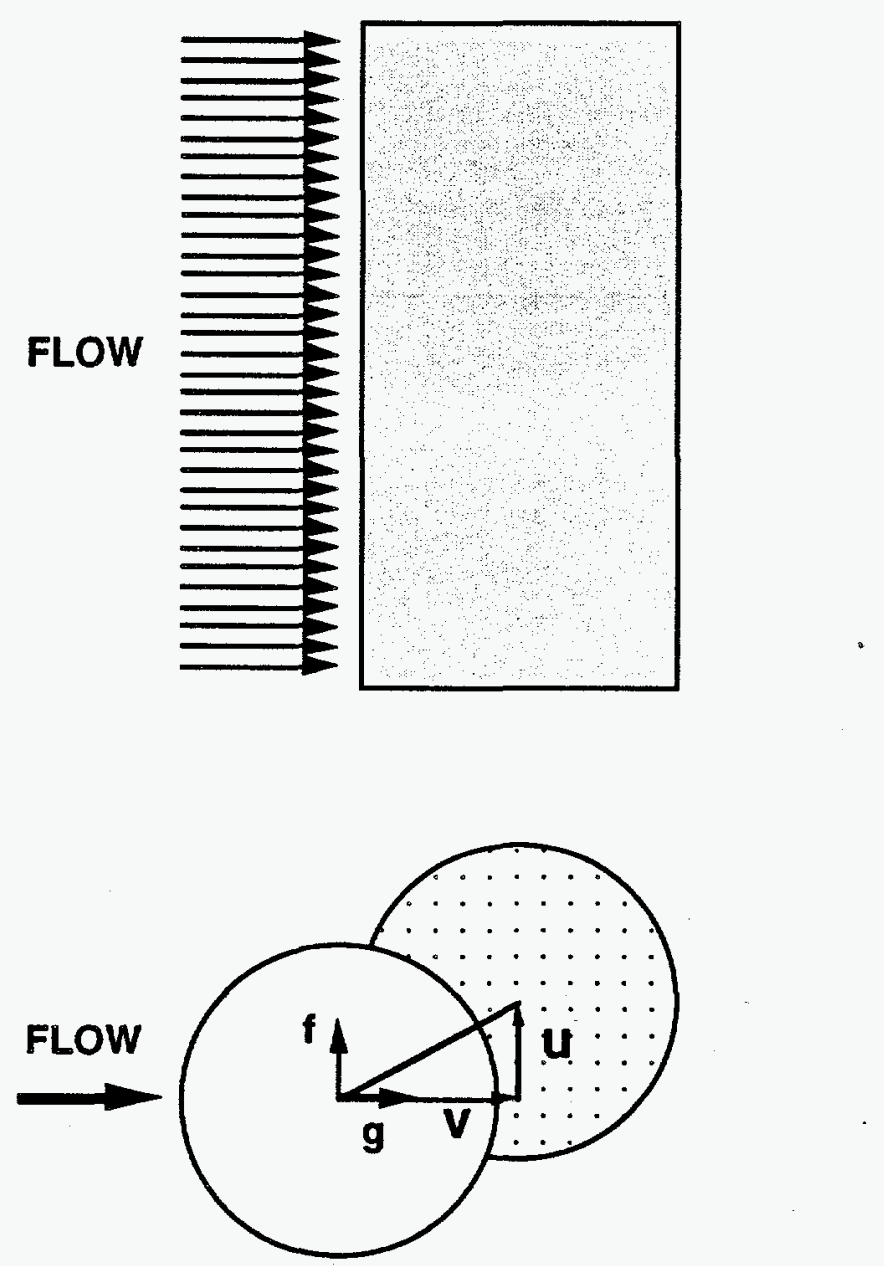

Fig. 4. Circular cylinder oscillating in crossflow 
where $\rho$ is fluid density, $R$ is cylinder radius, $t$ is time, and $\omega$ is circular frequency of cylinder oscillations. $\alpha, \beta, \sigma$, and $\tau$ are added-mass coefficients, $\alpha^{\prime}$, $\beta^{\prime}, \sigma^{\prime}$, and $\tau^{\prime}$ are fluid-damping coefficients, and $\alpha^{\prime \prime}, \beta^{\prime \prime}, \sigma^{\prime \prime}$, and $\tau^{\prime \prime}$ are fluidstiffness coefficients.

Various methods can be used to measure fluid-force coefficients. In this study, the unsteady-flow theory is used. Fluid-force coefficients can be determined by measuring the fluid forces acting on the cylinder as a result of its oscillations. If the cylinder is excited in the $\mathrm{x}$ direction, its displacement in the $\mathrm{x}$ direction is given by

$$
u=d_{0} \cos \omega t
$$

where $d_{0}$ is the oscillation amplitude. The motion-dependent fluid-force components acting on the cylinder in the $\mathrm{x}$ and $\mathrm{y}$ directions are

$$
\begin{aligned}
& f=\frac{1}{2} \rho U^{2} e_{\ell} \cos \left(\omega t+\phi_{\ell}\right) d_{o} \\
& g=\frac{1}{2} \rho U^{2} e_{d} \cos \left(\omega t+\phi_{d}\right) d_{0},
\end{aligned}
$$

where $e_{\ell}$ and $e_{\mathrm{d}}$ are the fluid-force amplitudes and $\phi_{\ell}$ and $\phi_{\mathrm{d}}$ are the phase angles by which the fluid forces acting on the cylinder lead the displacement of the cylinder.

By using Eqs. 1-3, we can also write the fluid-force components as

$$
\begin{aligned}
& f^{\prime}=\left(\rho \pi R^{2} \omega^{2} \alpha+\rho U^{2} \alpha^{\prime \prime}\right) d_{0} \cos \omega t-\rho U^{2} \alpha^{\prime} d_{0} \sin \omega t \\
& g=\left(\rho \pi R^{2} \omega^{2} \tau+\rho U^{2} \tau^{\prime \prime}\right) d_{o} \cos \omega t-\rho U^{2} \tau^{\prime} d_{0} \sin \omega t
\end{aligned}
$$

Use of Eqs. 4 and 6 and Eqs. 5 and 7 yields

$$
\begin{aligned}
& \alpha^{\prime \prime}=\frac{1}{2} e_{\ell} \cos \phi_{\ell}-\frac{\pi^{3}}{U_{r}^{2}} \alpha \\
& \tau^{\prime \prime}=\frac{1}{2} e_{d} \cos \phi_{d}-\frac{\pi^{3}}{U_{r}^{2}} \tau
\end{aligned}
$$




$$
\begin{aligned}
& \alpha^{\prime}=\frac{1}{2} e_{\ell} \sin \phi_{\ell} \\
& \tau^{\prime}=\frac{1}{2} e_{d} \sin \phi_{d},
\end{aligned}
$$

where $U_{r}$ is the reduced flow velocity $\left(U_{r}=\pi U / \omega R\right)$. The added mass coefficients $\alpha$ and $\tau$ can be calculated from the potential-flow theory (Chen 1987). Other fluidforce coefficients, $\beta^{\prime}, \beta^{\prime \prime}, \sigma^{\prime}$, and $\sigma^{\prime \prime}$, can be obtained in a similar manner by exciting the cylinder in the y direction.

\subsection{Equation of Motion for Vortex-Induced Stack Vibration}

A fluid/structure system with wind-induced vibration of a stack can be described by an unsteady-flow theory (Chen 1987; Chen et al. 1995). Once fluid excitation forces and motion-dependent fluid forces are known, the response of the stack to vortex-shedding-induced vibration can be predicted. The stack is subjected to a crossflow wind that is uniform along its length $\ell_{\mathbf{s}}$. The equation of motion in the lift direction is

$$
\begin{aligned}
E I \frac{\partial^{4} w(z, t)}{\partial z^{4}} & +C \frac{\partial w(z, t)}{\partial t}+m_{s} \frac{\partial^{2} w(z, t)}{\partial t^{2}}+\rho \pi R^{2} \alpha \frac{\partial^{2} w(z, t)}{\partial t^{2}}-\frac{\rho U^{2}}{\omega} \alpha^{\prime} \frac{\partial w(z, t)}{\partial t} \\
& -\rho U^{2} \alpha^{\prime \prime} w(z, t)=\frac{1}{2} \rho U^{2} D C_{L}^{\prime} \cos \left(\omega_{s} t\right)
\end{aligned}
$$

where $\mathrm{w}(\mathrm{z}, \mathrm{t})$ is the displacement of the stack in the lift direction, $\mathrm{D}$ is the stack diameter, $U$ is the wind speed, $\rho$ is the air density, $E I$ is the flexural rigidity, $C$ is the stack damping coefficient, $\mathrm{m}_{\mathrm{s}}$ is the stack mass per unit length, $\mathrm{C}_{\mathrm{L}}^{\prime}$ is the fluctuating lift coefficient, and $\omega_{\mathrm{S}}(=2 \pi \mathrm{SU} / \mathrm{D})$ is the circular frequency of vortex shedding. $\alpha, \alpha^{\prime}$, and $\alpha "$ are the added-mass, fluid-damping, and fluid-stiffness coefficients, respectively. All of the fluid-force coefficients are based on experimental data (Chen et al. 1993 and 1995). Figure 5 shows typical experimental data of fluid-damping and fluid-stiffness coefficients vs. reduced flow velocity.

Let

$$
w(z, t)=\sum_{n=1}^{\infty} D a_{n}(t) \Psi_{n}(z), \quad U_{r}=\frac{U}{f D}, \quad \gamma=\frac{\rho \pi D^{2}}{4 m_{s}}
$$



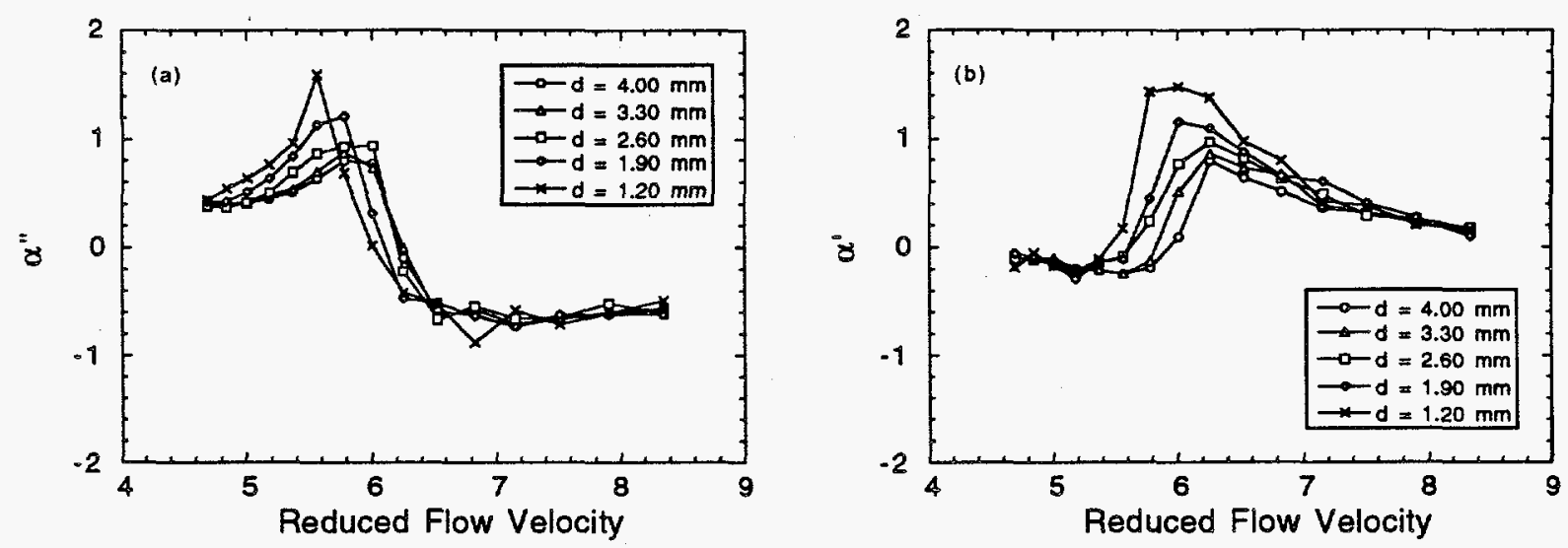

Fig. 5. Fluid-stiffness and fluid-damping coefficients for a single cylinder, $U=0.095 \mathrm{~m} / \mathrm{s}$ : (a) fluid-stiffness coefficient $\alpha^{\prime \prime}$; (b) fluid-damping coefficient $\alpha^{\prime}$.

where $\psi_{n}(z)$ is the $n$-th normal mode, $f$ is the oscillation frequency, and $U_{r}$ is reduced wind speed. Substituting Eqs. 10 in Eq. 9 yields

$$
\frac{d^{2} a_{n}}{d t^{2}}+2 \zeta \omega \frac{d a_{n}}{d t}+\omega^{2} a_{n}=\frac{C_{n}}{2(1+\gamma \alpha)}\left(\frac{\rho U^{2} C_{L}^{\prime} D}{m_{s}}\right) \cos \left(\omega_{s} t\right),
$$

where

$$
\begin{array}{ll}
\omega=\omega_{\mathrm{v}}\left(1+\gamma \mathrm{C}_{\mathrm{M}}\right)^{-0.5}, & \zeta=\frac{\zeta_{\mathrm{V}}}{1+\gamma \alpha}\left[\left(1+\gamma \mathrm{C}_{\mathrm{M}}\right)^{0.5}-\frac{\gamma \mathrm{U}_{\mathrm{r}}^{2} \alpha^{\prime}}{2 \zeta_{\mathrm{V}} \pi^{3}}\right], \\
\mathrm{C}_{\mathrm{M}}=\alpha+\frac{\mathrm{U}_{\mathrm{r}}^{2} \alpha^{\prime \prime}}{\pi^{3}}, & \mathrm{C}_{\mathrm{n}}=\frac{1}{\ell_{\mathrm{S}}} \int_{0}^{\ell_{\mathrm{s}}} \Psi_{\mathrm{n}}(\mathrm{z}) \mathrm{dz} .
\end{array}
$$

Note that $\omega$ and $\zeta$ are the circular frequency and modal damping ratio, respectively, for the stack in crosswind flow. $C_{M}$ is called an added-mass coefficient; when $U_{r}=0$, it is equal to $\alpha$. When $U_{r} \neq 0, C_{M}$ depends on both $U_{r}$ and $\alpha^{\prime \prime}$, which in turn, depends on $U_{\mathrm{r}}$ and the stack oscillation amplitude. $\omega_{\mathrm{v}}$ and $\zeta_{\mathrm{v}}$ are the in-vacuum natural frequency and modal damping ratio, respectively (Chen et al. 1995). 


\subsection{Lock-In Resonance of Stack}

As indicated by Chen and Cai (1992), the stack was excited by vortex shedding. When the wind speed was $>15 \mathrm{~m} / \mathrm{s}$, the stack was in the synchronization region called lock-in resonance. Figure 6 shows the vortexshedding frequency as a function of wind speed $U$ in the range of $U=7-25 \mathrm{~m} / \mathrm{s}$ when the Strouhal number is $0.150,0.175$, and 0.200 . It is noted that the vortexshedding frequencies in this wind speed range are close to the first four natural frequencies of the stack (see Fig. 6). This is one of the requirements for lock-in resonance. The mass-damping parameter is 2, which is less than 32 (Chen and Cai 1992). This is the second requirement for lock-in resonance.

The stack response due to vortex shedding can be obtained by solving Eq. 11 . Note that because the fluid-force coefficients $\alpha^{\prime}$ and $\alpha^{\prime \prime}$ are a function of $U_{r}$, the natural frequency $\omega$ and modal damping ratio $\zeta$ are a function of the reduced flow velocity $U_{r}$.

Consider a stack with the mass ratio $\gamma$ of 0.2 and damping ratio $\xi_{\mathrm{v}}$ of $2 \%$, a Strouhal number $S$ of 0.175 , and a fluctuating lift coefficient $\mathrm{C}_{\mathrm{L}}^{\prime}$ of 0.5 . The root mean square (RMS) values of the four-point stack motions (corresponding to the ends of the four guy wires) in the lift direction were plotted as the function of wind speed in Fig. 7.

For the third mode, lock-in resonance occurs at $U \approx 15 \mathrm{~m} / \mathrm{s}$, and Point 2 $(45.7 \mathrm{~m})$, associated with the second-level guy wire, exhibits the largest oscillating amplitude. From this observation, it may be concluded that the vibration mode was about the same as the mode shape of the third mode (Fig. 3c). Because the upper portion of the stack included spoilers and the lower portion did not, this particular mode was vulnerable to vortex-shedding-induced resonance due to a large participation factor associated with vortex shedding (Chen and Cai 1992).

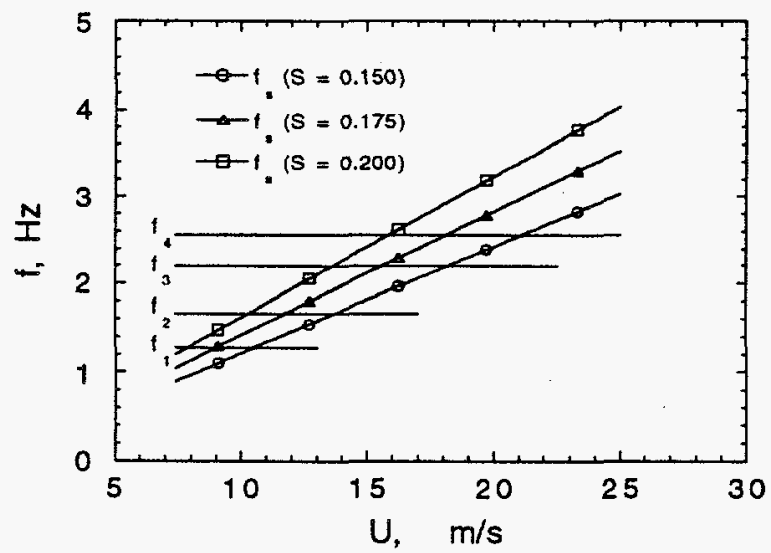

Fig. 6. Vortex-shedding frequencies as a function of wind speed 

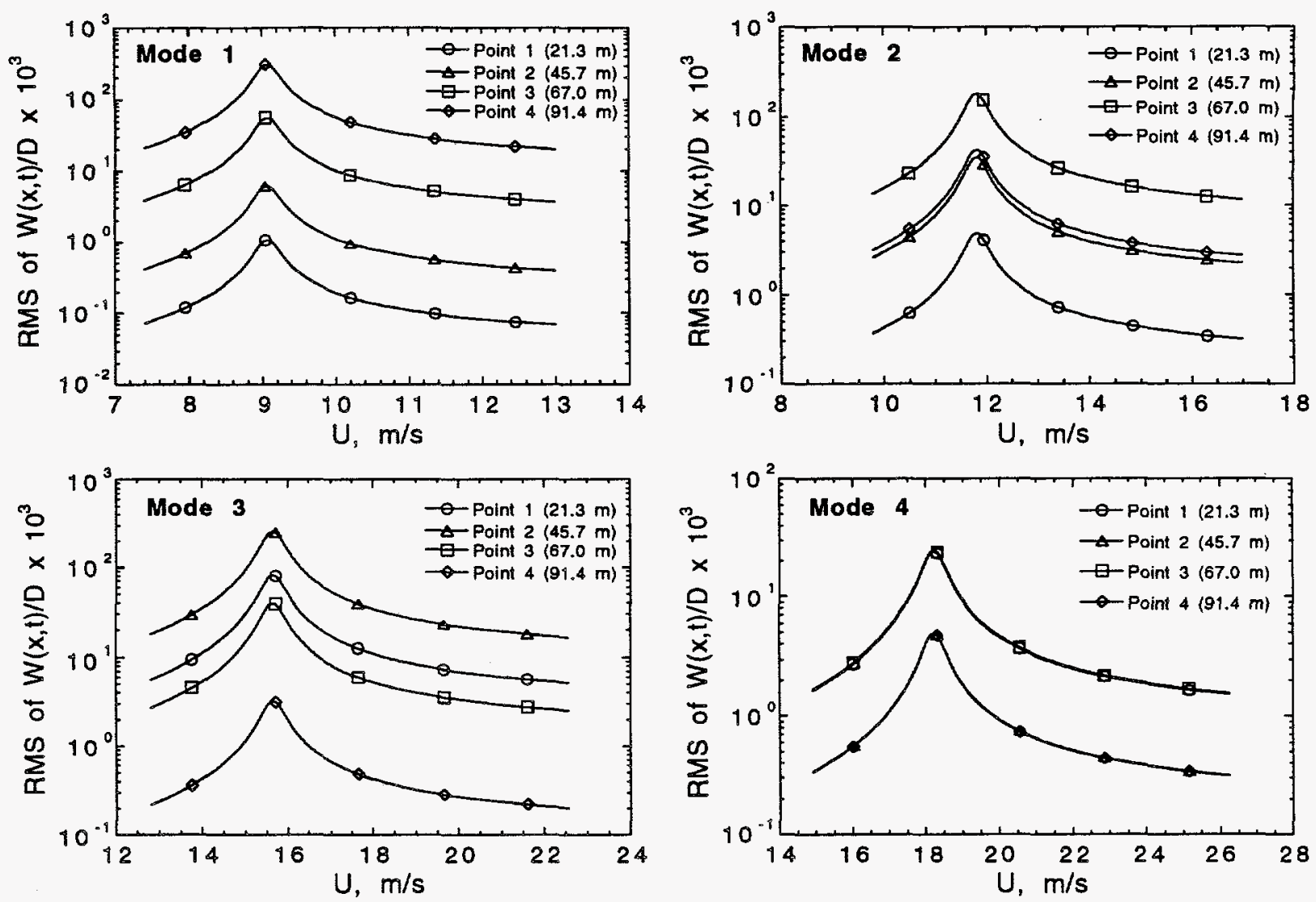

Fig. 7. RMS values of stack motion as a function of wind speed

From the calculation (Fig. 7) and the observation, we concluded again that the stack vibration was excited by vortex shedding at the lower portion associated with the third mode of the stack.

\section{Parametric and External Resonances of Nonlinear Cables}

\subsection{Literature Review}

Nonlinear free vibration of cables is the topic of investigation by Rega et al. (1984), Luongo et al. (1984), and Takahashi and Konishi (1987). Forced vibration of elastic suspended cables was studied by Al-Noury and Ali (1985), Benedettini and Rega (1987), and Rao and Iyengar (1991). Al-Noury and Ali (1985) investigated the undamped, nonplanar response associated with internal resonance under harmonic excitation in either the vertical or transverse direction. Benedettini and Rega (1987) studied nonlinear, in-plane vibration of a cable under harmonic 
excitation when both quadratic and cubic nonlinear terms were present. Considering both internal and external resonances, Rao and Iyengar (1991) studied the coupled nonlinear cable in both in-plane and out-of-plane vibration. However, very little has appeared in the literature that reports work on parametric and external resonances of suspended cables. Narasimha (1968) studied the stability problem of nonplanar motion of the cable. Takahashi (1991) studied the stability of a cable under an axial sinusoidally time-varying load, and discussed, in detail, the unstable regions of the parametric and combination resonances. However, both Narasimha (1968) and Takahashi (1991) neglected nonlinear effects on parametric and external resonances. Perkins (1992) derived a nonlinear model of a suspended elastic cable with small tangential oscillations of one support. In this model, he utilized a first-order perturbation approach to analyze modal interactions in the nonlinear response of elastic cables under parametric and external excitation, and his experimental measurements were in good qualitative agreement with theoretical predictions.

\subsection{Nonlinear System of Elastic Cables}

One objective of this study is to investigate the nonlinear dynamics and instability of guy wires for practical applications. The guy wires in the original system (see Fig. 1) can be described as heavy nonlinear elastic cables with cubic nonlinearities due to cable stretching and quadratic nonlinearities due to equilibrium cable curvature in a tilted configuration. The support at the upper end of the cable is also subject to a pulsating displacement associated with the bending vibration of the stack due to vortex shedding.

Because large-amplitude out-of-plane vibration of the wire was not observed from the stack/wire response (Chen and Cai 1992), only an in-plane-motion model of cable vibration was considered in this study. In some cases, depending on the cable tension and other parameters, coupled in-plane and out-of-plane motion may be important. In the present work, we derived a nonlinear model of in-plane motion of a heavy elastic cable. This model includes cubic and quadratic nonlinearities, and a pulsating excitation at the support at the upper end of the cable due to lock-in resonance of the stack. Axial motion at the support leads to parametric excitation, whereas transverse motion contributes to both parametric and external excitations. Therefore, the tilted angle of the cable, which determines the ratio of axial and transverse motions, is very important to the parametric and external resonances.

A particular static-equilibrium configuration of the cable, a tilted configuration based on the original system shown in Fig. 1, was introduced into the dynamic model. This static deformation significantly affected the initial 
curvature of the cable as a quadratic nonlinearity, and thus could give rise to new phenomena.

Numerical analysis was conducted (Cai and Chen 1994) to investigate the nonlinear dynamics of the cable under parametric and external resonances. When the system is simultaneously affected by a parametric excitation term and nonlinear terms, numerical simulation in time series provides a better understanding of the phenomena than the classical perturbation analysis approach. After introducing consistent simplifying assumptions, the partial differential equations of planar motion were reduced to a system of coupled equations via a Galerkin procedure by assuming a modal deflection shape. Thus, coupling effects of different modes were considered in nonlinear terms of the cable.

Even though the work reported here studied a specific nonlinear instability problem of cables supporting a stack, the methodology used can be applied to general problems of parametric excitation of elastic cables that are suspended with shallow sag and inclination.

\subsection{Equation of Motion for an Elastic Cable}

Given the original system in Fig. 1, a guy wire can be described as a heavy elastic cable suspended between two supports in a tilted configuration (see Fig. 8 for a representation of a guy wire at the second level from the bottom). The lower end of the cable is fixed to the ground and the upper end is pin-supported and movable horizontally to simulate the bending motion of the stack due to vortex shedding. The motion at the support at the upper end can be simplified to a sinusoidal displacement in the horizontal direction, i.e.,

$$
\mathrm{w}=\mathrm{w}_{0} \sin \omega_{\mathrm{s}} \mathrm{t},
$$

where $w_{0}$ is excitation amplitude and $\omega_{\mathrm{s}}$ is excitation frequency. Thus, the motion components $\mathrm{u}$ and $\mathrm{v}$ at the support at the upper end in both axial and transverse directions of the cable are

$$
\begin{aligned}
& u(\ell, t)=u_{0} \sin \omega_{s} t=-w_{0} \cos \alpha \sin \omega_{s} t \\
& v(\ell, t)=v_{0} \sin \omega_{s} t=w_{0} \sin \alpha \sin \omega_{s} t
\end{aligned}
$$

where $\ell$ is the distance between the two supports and $\alpha$ is the tilt angle between the cable and the ground. 


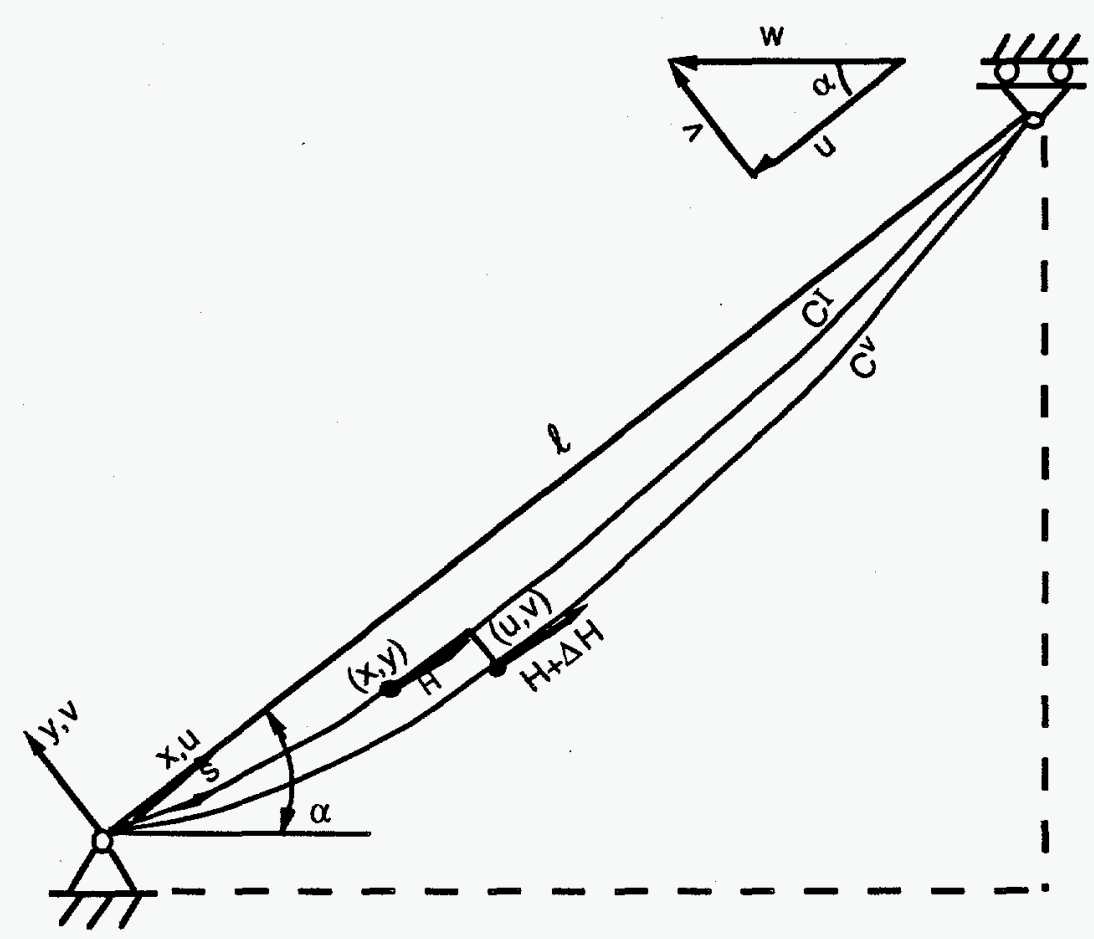

Fig. 8. Cable supported in angled configuration

The initial static-equilibrium configuration $\mathrm{C}^{\mathrm{I}}$ in Fig. 8 lies in the OXY plane and is represented by the function $y(s)$, $s$ being a curvilinear abscissa. Let $E, A$, and $m$ be the elastic modulus, the cross-sectional area, and the mass per unit length of the cable, respectively. The varied configuration $\mathrm{C}^{\mathrm{v}}$ can be described by the displacement coordinate $\mathrm{u}(\mathrm{s}, \mathrm{t})$ and $\mathrm{v}(\mathrm{s}, \mathrm{t})$ (see Fig. 8).

By assuming the Lagrangian strain as the strain measure, the extensional strain in the cable motion is

$$
\varepsilon(s, t)=\frac{H}{E A}+\frac{\partial x}{\partial s} \frac{\partial u}{\partial s}+\frac{\partial y}{\partial s} \frac{\partial v}{\partial s}+\frac{1}{2}\left[\left(\frac{\partial u}{\partial s}\right)^{2}+\left(\frac{\partial v}{\partial s}\right)^{2}\right]
$$

If we assume that $\mathrm{ds} \approx \mathrm{dx}$ (shallow sag), and that the cable tension in the initial configuration is equal to the longitudinal component of the initial tension $\mathrm{H}$, the initial strain is negligible with respect to unity, which leads to the assumption that $\mathrm{H} / \mathrm{EA} \ll 1$. The gradient of the longitudinal component of the dynamic displacement is negligible to unity. Thus, the static equilibrium configuration of the cable in Fig. 8 can be derived easily; the result is 


$$
y(x)=\operatorname{ctg} \alpha\left[\frac{\ell n\left(1+\frac{m g \ell}{\mathrm{H}} \sin \alpha \frac{\mathrm{x}}{\ell}\right)}{\ell \mathrm{n}\left(1+\frac{\mathrm{mg} \ell}{\mathrm{H}} \sin \alpha\right)}-\frac{\mathrm{x}}{\ell}\right] \ell_{\text {. }}
$$

Let $\tilde{y}=y / \ell, \tilde{x}=x / \ell$, and $\beta=\frac{m g \ell}{H}$, and Eq. 17 becomes

$$
\tilde{\mathbf{y}}(\tilde{\mathbf{x}})=\operatorname{ctg} \alpha\left[\frac{\ln (1+\beta \sin \alpha \tilde{\mathbf{x}})}{\ell \ln (1+\beta \sin \alpha)}-\tilde{\mathbf{x}}\right]
$$

and

$$
\frac{d \tilde{y}}{d \tilde{x}}=\operatorname{ctg} \alpha\left[\frac{\beta \sin \alpha}{(1+\beta \sin \alpha \tilde{x}) \ln (1+\beta \sin \alpha)}-1\right] .
$$

The coupled equations of motion of the cable are

$$
\begin{aligned}
& \frac{\partial}{\partial x}\left\{E A\left[\frac{\partial u}{\partial x}+\frac{d y}{d x} \frac{\partial v}{\partial x}+\frac{1}{2}\left(\frac{\partial v}{\partial x}\right)^{2}\right]\right\}=m \ddot{u} \\
& \frac{\partial}{\partial x}\left\{H \frac{\partial v}{\partial x}+E A\left(\frac{d y}{d x}+\frac{\partial v}{\partial x}\right)\left[\frac{\partial u}{\partial x}+\frac{d y}{d x} \frac{\partial v}{\partial x}+\frac{1}{2}\left(\frac{\partial v}{\partial x}\right)^{2}\right]\right\}=m \ddot{v}+c \dot{v},
\end{aligned}
$$

where $u$ and $v$ are longitudinal and transverse components, respectively (Fig. 8), and $\mathrm{c}$ is the viscous damping coefficient per unit length of transverse motion.

It is possible to reduce the two coupled differential equations to one equation (Luongo et al. 1984). The longitudinal inertia force mü can be neglected in Eq. 20, to give

$$
\frac{\partial u}{\partial x}+\frac{d y}{d x} \frac{\partial v}{\partial x}+\frac{1}{2}\left(\frac{\partial v}{\partial x}\right)^{2}=h(t)
$$

According to Eq. 22, the additional cable tension $\Delta \mathrm{H}=\mathrm{EA} \cdot \mathrm{h}(\mathrm{t})$ is a function of time only. It follows that

$$
u(x, \hat{t})=h(t) x-\int_{0}^{x}\left[\frac{d y}{d x} \frac{\partial v}{\partial x}+\frac{1}{2}\left(\frac{\partial v}{\partial x}\right)^{2}\right] d x,
$$


where the value of the constant $h(t)$ is determined by the boundary condition $u(\ell, t)=u_{0} \sin \omega_{s} t$ of the cable at the upper end (Eq. 14); thus

$$
h(t)=\frac{1}{\ell}\left\{u_{0} \sin \omega_{s} t+\int_{0}^{\ell}\left[\frac{d y}{d x} \frac{\partial v}{\partial x}+\frac{1}{2}\left(\frac{\partial v}{\partial x}\right)^{2}\right] d x\right\} .
$$

Using Eqs. 22 and 24, we can reduce Eqs. 20 and 21 to one equation, i.e., the motion can be described by the unique partial integro-differential equation in the transverse displacement component

$$
\frac{\partial}{\partial x}\left(H \frac{\partial v}{\partial x}+E A\left(\frac{d y}{d x}+\frac{\partial v}{\partial x}\right)\left\{\frac{u_{0}}{\ell} \sin \omega_{s} t+\frac{1}{\ell} \int_{0}^{\ell}\left[\frac{d y}{d x} \frac{\partial v}{\partial x}+\frac{1}{2}\left(\frac{\partial v}{\partial x}\right)^{2}\right] d x\right\}\right)=m \ddot{v}+c \dot{v}
$$

A nondimensional form of the equation of motion is obtained by introducing the following normalized variables:

$$
\begin{aligned}
& \mu=\frac{E A}{H}, \\
& \tilde{\mathbf{x}}=\mathrm{x} / \ell, \quad \tilde{\mathrm{y}}=\mathrm{y} / \ell, \\
& \tilde{\mathbf{u}}=\mathrm{u} / \ell, \quad \tilde{\mathbf{u}}_{0}=\mathrm{u}_{0} / \ell, \\
& \tilde{\mathrm{v}}=\mathrm{v} / \ell, \quad \tilde{\mathbf{v}}_{0}=\mathrm{v}_{0} / \ell, \\
& \tilde{\mathrm{t}}=\omega_{1} \mathrm{t},
\end{aligned}
$$

and

$$
\left.\begin{array}{l}
\omega_{\mathrm{n}}=\frac{\mathrm{n} \pi}{\ell} \sqrt{\frac{\mathrm{H}}{\mathrm{m}}} \\
\zeta_{\mathrm{n}}=\frac{\mathrm{c}}{2 \mathrm{~m} \omega_{\mathrm{n}}}
\end{array}\right\} \mathrm{n}=1,2,3, \ldots
$$

where $\omega_{n}$ is the frequency and $\zeta_{n}$ is the damping ratio of the cable in the $n$-th mode. 
Using Eqs. 26 and 27 and omitting the tilde, we can rewrite the equation of motion as

$$
\begin{aligned}
\frac{\partial}{\partial x}\left(\frac{\partial v}{\partial x}\right. & \left.+\mu\left(\frac{d y}{d x}+\frac{\partial v}{\partial x}\right)\left\{u_{0} \sin \frac{\omega_{s}}{\omega_{1}} t+\int_{0}^{1}\left[\frac{d y}{d x} \frac{\partial v}{\partial x}+\frac{1}{2}\left(\frac{\partial v}{\partial x}\right)^{2}\right] d x\right\}\right) \\
& =n^{2} \pi^{2}\left[\left(\frac{\omega_{1}}{\omega_{n}}\right)^{2} \ddot{v}+2 \zeta_{n}\left(\frac{\omega_{1}}{\omega_{n}}\right) \dot{v}\right]
\end{aligned}
$$

Let

$$
v(x, t)=v_{1}+v_{2}=\sum_{n=1}^{\infty} q_{n}(t) \phi_{n}(x)+x v_{0} \sin \frac{\omega_{s}}{\omega_{1}} t
$$

where $x=1, v_{2}\left[=x v_{0} \sin \left(\omega_{s} / \omega_{1}\right) t\right]$ corresponds to the boundary condition at the support at the upper end (see Eq. 15), $q_{n}(t)$ is the generalized transverse displacement of the cable, and $\phi_{n}(x)$ is the modal function and can be simply described as

$$
\phi_{\mathbf{n}}(\mathrm{x})=\sqrt{2} \sin \mathrm{n} \pi \mathrm{x}
$$

and

$$
\int_{0}^{1} \phi_{\mathrm{n}}^{2}(\mathrm{x}) \mathrm{dx}=1
$$

Then, by applying the Galerkin method to Eq. 28, a system of ordinary differential equations is obtained.

$$
\begin{aligned}
\ddot{q}_{n}+2 \zeta_{n} n \dot{q}_{n}+n^{2}\left\{1+\mu\left[\frac{1}{4} v_{0}^{2}\left(1-\cos 2 \omega_{r} t\right)+\left(u_{0}+I_{0} v_{0}\right) \sin \omega_{r} t\right]\right\} q_{n} \\
+\mu n^{2}\left[I_{6 n}-\frac{1}{n \pi}\left(I_{2 n} v_{0} \sin \omega_{r} t+I_{7 n}\right)\right] q_{n} \sum_{n=1}^{\infty} q_{n}+\mu n^{2} I_{3 n} q_{n} \sum_{n=1}^{\infty} q_{n}^{2} \\
\quad-\frac{\mu}{\pi^{2}}\left(I_{1 n} v_{0} \sin \omega_{r} t+I_{5 n}\right)\left(I_{2 n} v_{0} \sin \omega_{r} t+I_{7 n}\right) \sum_{n=1}^{\infty} q_{n} \\
=\omega_{r}^{2} I_{4 n} v_{0} \sin \omega_{r} t-2 \zeta_{n} n \omega_{r} I_{4 n} v_{0} \cos \omega_{r} t \quad n=1,2,3, \ldots,
\end{aligned}
$$


where

$$
\begin{aligned}
& \frac{\omega_{\mathrm{n}}}{\omega_{1}}=\mathrm{n}, \\
& \frac{\omega_{\mathrm{s}}}{\omega_{1}}=\omega_{\mathrm{r}}=f_{\mathrm{r}},
\end{aligned}
$$

and $I_{0}, I_{j n}(j=1,2,3, \ldots 7)$ are defined in the Appendix. Equation 32 can be written as

$$
\begin{aligned}
\ddot{q}_{n}+2 \zeta_{n} n \dot{q} & +n^{2}\left(C_{0} q_{n}+C_{1 n} \sum_{n=1}^{\infty} q_{n}+C_{2 n} q_{n} \sum_{n=1}^{\infty} q_{n}+C_{3 n} q_{n} \sum_{n=1}^{\infty} q_{n}^{2}\right) \\
& =A_{n} \sin \omega_{r} t+B_{n} \cos \omega_{r} t \quad n=1,2,3, \ldots,
\end{aligned}
$$

where $C_{0}, C_{1 n}, C_{2 n}, C_{3 n}, A_{n}$, and $B_{n}$ are defined in the Appendix.

If the tilt angle $\alpha$ is small and the excitation amplitude $w_{0}$ is large, the parametric and external resonance will be significant and some nonlinear coupling effects can be neglected. Then, the equation of motion can be simplified to

$$
\begin{aligned}
\ddot{q}_{n}+2 \zeta_{n} n \dot{q}_{n}+ & n^{2}\left[\left(1+e \sin \omega_{r} t\right) q_{n}+C_{2 n} q_{n}^{2}+C_{3 n} q_{n}^{3}\right] \\
& =A_{n} \sin \omega_{r} t+B_{n} \cos \omega_{r} t \quad n=1,2,3, \ldots,
\end{aligned}
$$

where $\mathrm{C}_{2 \mathrm{n}}$ and $\mathrm{C}_{3 \mathrm{n}}$ are coefficients for quadratic and cubic nonlinear terms, respectively. In Eq. 36, e, the excitation parameter, is expressed as

$$
\mathrm{e} \approx \mu\left(\mathrm{u}_{0}+\mathrm{I}_{0} \mathrm{v}_{0}\right) \approx \mu \mathrm{u}_{0}
$$

\subsection{Parametric Resonance of Cable}

The parametric resonance of the guy wires in this case was unique. The mechanism was given further consideration to reconfirm the predicted mechanism.

Equation 36 can be rewritten as 


$$
\begin{aligned}
& \ddot{q}+2 \xi_{w} \omega_{w} \dot{q}+\omega_{w}^{2}\left[1+e \cos \left(\omega_{s} t\right)\right] q=0, \\
& \omega_{w}=2 \pi f_{w}
\end{aligned}
$$

and

$$
\omega_{\mathrm{s}}=2 \pi \mathrm{f}_{\mathrm{s}},
$$

where $q$ is the generalized coordinate for the first mode of the wire, $f_{w}$ is the fundamental natural frequency of the wire, $f_{s}$ is the stack oscillation frequency, $\xi_{\mathrm{w}}$ is the modal damping ratio, and $\mathrm{e}$, the excitation parameter, is given by

$$
e=(E A / T)(d / L) \cos \alpha,
$$

where $\mathrm{T}$ is the wire tension, $\mathrm{EA}$ is the modulus of elasticity multiplied by the cross-sectional area of the wire, $d$ is the stack oscillation amplitude at the upper end of the wire, $L$ is the wire length, and $\alpha$ is the angle between the wire and the ground level.

Equation 38 is the well-known Mathieu equation. When certain conditions on $f_{w}, f_{s}$, and $e$ are met, the trivial solution of $q=0$ is unstable and the response of $q$ is unbounded until other nonlinear effects become important. The instability in this case is called parametric resonance (Chen 1987; Stoker 1950).

It is assumed that the value of $e$ is very small and that $f_{w} \approx f_{s} / 2$. The first approximation for the solution of Eq. 38 is

$$
q=\operatorname{acos}\left(\frac{\omega_{\mathrm{s}}}{2} t+\theta\right),
$$

where $a$ and $\theta$ are given by

$$
\begin{aligned}
& \dot{\mathrm{a}}=-\mathrm{a} \zeta_{\mathrm{w}} \omega_{\mathrm{w}}-\frac{\mathrm{ae} \omega_{\mathrm{w}}^{2}}{2 \omega_{\mathrm{s}}} \sin 2 \theta, \\
& \dot{\theta}=\omega_{\mathrm{w}}-\frac{\omega_{\mathrm{S}}}{2}-\frac{\mathrm{e} \omega_{\mathrm{w}}^{2}}{2 \omega_{\mathrm{s}}} \cos 2 \theta
\end{aligned}
$$


If we let

$$
\mathbf{u}=\mathbf{a} \cos \theta,
$$

and

$$
\mathrm{v}=\mathbf{a} \sin \theta
$$

Eqs. 41 are reduced to the form

$$
\begin{aligned}
& \dot{\mathrm{u}}=-\zeta_{\mathrm{w}} \omega_{\mathrm{w}} \mathrm{u}-\left[\frac{\mathrm{e} \omega_{\mathrm{w}}^{2}}{2 \omega_{\mathrm{s}}}+\left(\omega_{\mathrm{w}}-\frac{\omega_{\mathrm{s}}}{2}\right)\right] \mathrm{v}, \\
& \dot{\mathrm{v}}=-\left[\frac{\mathrm{e} \omega_{\mathrm{w}}^{2}}{2 \omega_{\mathrm{s}}}-\left(\omega_{\mathrm{w}}-\frac{\omega_{\mathrm{s}}}{2}\right)\right] \mathrm{u}-\zeta_{\mathrm{w}} \omega_{\mathrm{w}} \mathrm{v} .
\end{aligned}
$$

The system is autonomous and its characteristic equation is

$$
\mathrm{s}^{2}+2 \zeta_{\mathrm{w}} \omega_{\mathrm{w}} \mathrm{S}+\left[\left(\zeta_{\mathrm{w}} \omega_{\mathrm{w}}\right)^{2}-\left(\frac{\mathrm{e} \omega_{\mathrm{w}}^{2}}{2 \omega_{\mathrm{s}}}\right)+\left(\omega_{\mathrm{w}}-\frac{\omega_{\mathrm{s}}}{2}\right)^{2}\right]=0
$$

The roots of the characteristic equation (Eq. 44) are

$$
\mathrm{S}_{1,2}=-\zeta_{\mathrm{w}} \omega_{\mathrm{w}} \pm \sqrt{\left(\frac{\mathrm{e} \omega_{\mathrm{w}}^{2}}{2 \omega_{\mathrm{s}}}\right)^{2}-\left(\omega_{\mathrm{w}}-\frac{\omega_{\mathrm{s}}}{2}\right)^{2}}
$$

If $S$ has a positive real component, $a$ and $q$ increase exponentially. To determine the interval of the parametric resonance, we set $S=0$. Then, the interval of the parametric resonance in the first approximation is as follows:

$$
4-\sqrt{\mathrm{e}^{2}-16 \zeta_{\omega}^{2}}<\left(\frac{\omega_{\mathrm{s}}}{\omega_{\mathrm{w}}}\right)^{2}<4+\sqrt{\mathrm{e}^{2}+16 \zeta_{\omega}^{2}} .
$$

The frequency width $\Delta \omega$ of the parametric resonance zone is

$$
\Delta \omega=2\left[\left(\sqrt{4+\sqrt{\mathrm{e}^{2}-16 \zeta_{\omega}^{2}}}\right)-2\right] \omega_{\mathrm{w}} .
$$


In Eqs. 46 and 47 , if $\mathrm{e} \geq 4 \zeta_{\omega}$, parametric resonance exists.

The results for parametric resonance with the Mathieu equation (Eq. 38) are shown in Fig. 9. For differing damping ratios, the principal instability regions are functions of excitation parameter e in Fig. 9.

The detailed instability regions for all wires can be calculated with Eqs. 39 and 46. To predict instability regions accurately, the properties of the guy wires must be known precisely, e.g., the values of damping and the modulus of helically woven wires must be known. Because no measurements were made, it was necessary to estimate all of the parameters.

Consider the wire at the $45.7 \mathrm{~m}$ level when its tension is between 2710 and $2970 \mathrm{~kg}$. The natural frequency of the wire $f_{\omega}$ is between 1.07 and $1.12 \mathrm{~Hz}$ and the dominant frequency of the stack, the third mode of Model $1, f_{s}$, is $2.20 \mathrm{~Hz}$ (Fig. 3). The value of $f_{s} / f_{\omega}=1.96-2.04$; the stack oscillation frequency is approximately twice that of the wire. The excitation parameter corresponding to the stack oscillation amplitude of $\approx 2.5 \mathrm{~cm}$ is calculated to be $\approx 0.15$. With these parameters, the wire will be in the unstable region (see Fig. 9) if the damping ratio is a few percent. This means that the wire oscillations are associated with the parametric resonance due to vortex shedding of the stack.

As shown in Fig. 9, with a given damping ratio $\zeta$, a finite critical value of excitation parameter $e$ is required for parametric instabilities. If $e$ is less than the critical value, the cable is not subjected to parametric instabilities.

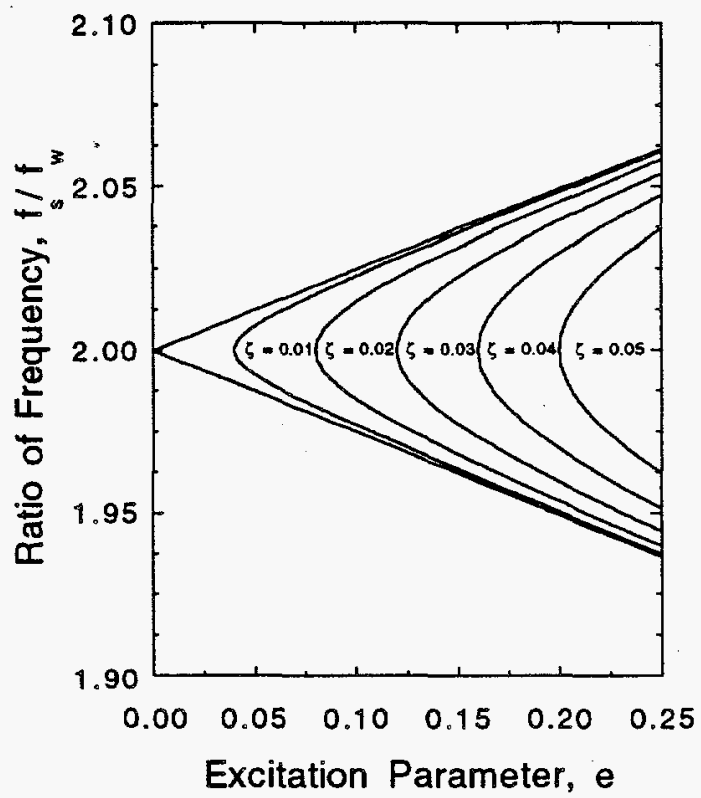

Fig. 9. Unstable region of principal parametric resonance 


\subsection{Numerical Simulation for Parametric and External Resonances}

We notice that the dynamics of an elastic suspended cable with both cubic and quadratic nonlinearities under parametric and combination resonance are very difficult to resolve by pure analytical means. Therefore, a new approach to the numerical solution of cable dynamics is introduced in this study (Cai and Chen 1994). Numerical simulations of the nonlinear dynamics of the suspended cable described in Fig. 8 are based on the fourth-order Runge-Kutta method of integrating the five coupled equations (see Eq. 35, $\mathrm{n}=1,2,3,4,5$ ) that represent a multiple-degree-of-freedom system for the cable and take into account the coupling effects of nonlinearities and parametric and combination instabilities. The focus of this study was on steady-state cable vibrations. Thus, numerical integrations were performed with a given arbitrary set of initial conditions for a relatively long time to ensure that transient effects had died out before the output was examined.

Parameters and material properties of the cable used in the numerical simulation are based on the original system as shown in Fig. 1 and described in Tables 1 and 2. To analyze the dynamic response of the cable under parametric and external resonances, system parameters, such as excitation frequency ratio $\omega_{\mathrm{r}}\left(\mathrm{f}_{\mathrm{r}}\right)$, excitation amplitude $\mathrm{w}_{0}$, tilt angle $\alpha$, and damping ratio $\zeta$, were chosen for study.

Figure 10 gives the RMS displacements of the cable at $x=0.500$ and $x=0.125$ versus the excitation frequency ratio $f_{r}$ with various excitation amplitudes $w_{0}$. Regardless of the values of the excitation amplitudes, the cable was subjected to external resonance at $f_{r}=1.0,2.0,3.0$, and 4.0 (see Fig. 10b). At $x=0.500$ (Fig. 10a), the second and the fourth mode responses are expected to be zero if they are due to external resonance only. When the excitation amplitude $\mathrm{w}_{0}$ is $>0.0002$ and $f_{r}=2.0$ and 4.0, parametric resonance exists, corresponding to the first and second primary parametric instability frequencies, i.e., primary parametric instabilities occur near $\omega_{\mathrm{s}}=2 \omega_{1}$ and $\omega_{\mathrm{s}}=2 \omega_{2}$. These phenomena can be viewed clearly from the maximum cable responses shown in Figs. 11a-d, for $f_{r}=1.0,2.0$, 3.0, and 4.0. For $f_{r}=1.0$ and 3.0, it appears that only external resonances dominate the cable motion, with $\omega_{\mathrm{s}}=\omega_{1}$ and $\omega_{\mathrm{s}}=\omega_{3}$, respectively (see Figs. 11a and 11c). However, for $\mathrm{f}_{\mathrm{r}}=2.0$ and 4.0, when excitation amplitude $\mathrm{w}_{0}=0.0001$ and 0.0002 , external resonances dominate cable motion, with $\omega_{\mathrm{s}}=\omega_{2}$ and $\omega_{\mathrm{s}}=\omega_{4}$, corresponding to the second and forth modal shapes; when excitation amplitude increases to $\mathrm{w}_{0}=0.0003$ and 0.0004 , parametric resonances dominate the cable motion, with $\omega_{\mathrm{s}}=2 \omega_{1}$ and $\omega_{\mathrm{s}}=2 \omega_{2}$, corresponding to the first and second modal shapes (see Figs. 11b and 11d). It is obvious that, with further increase in excitation amplitude $\mathrm{w}_{0}$, the parametric resonance characteristics of cable motion with $\mathrm{f}_{\mathrm{r}}=2.0$ and 4.0 will become outstanding and the effects of external resonance may be neglected. 

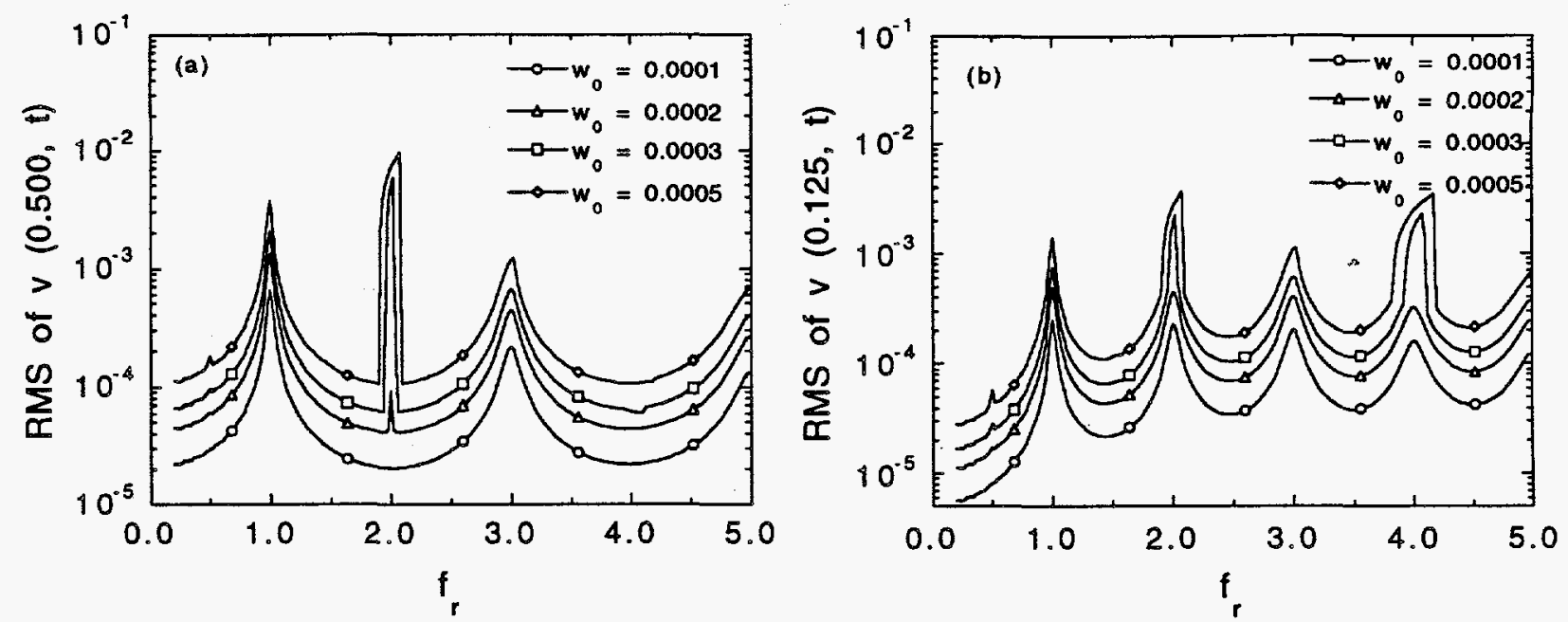

Fig. 10. RMS displacements of cable at (a) $x=0.500$ and (b) $x=0.125$ versus excitation frequency ratio $f_{r}$ with various excitation amplitudes $w_{0}$ $(\alpha=0.2 \pi, \beta=0.02, \zeta=0.02, \mu=500)$
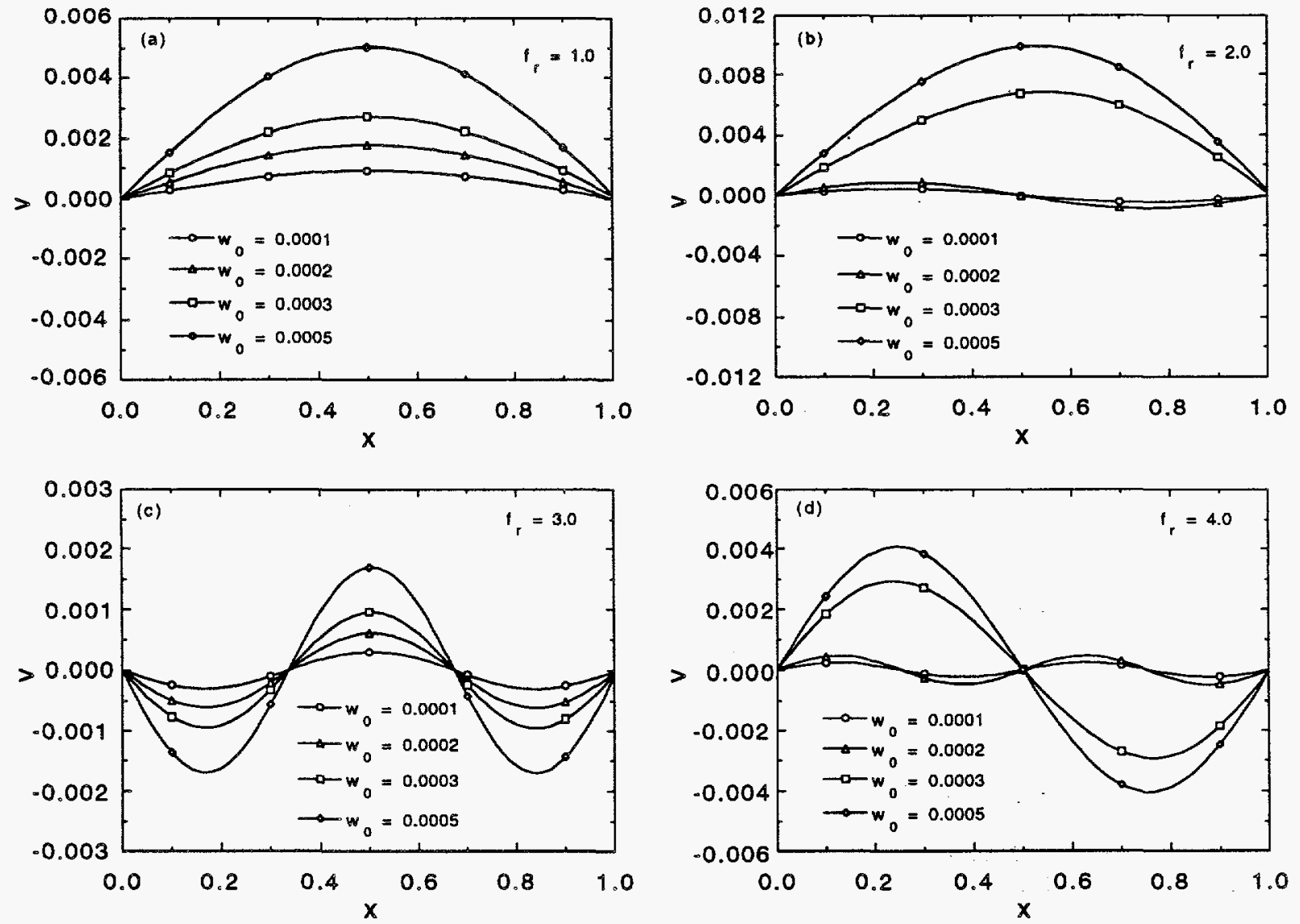

Fig. 11. Maximum cable responses with various excitation amplitudes $w_{0}(\alpha=$ $0.2 \pi, \beta=0.02, \zeta=0.02, \mu=500$ ) 

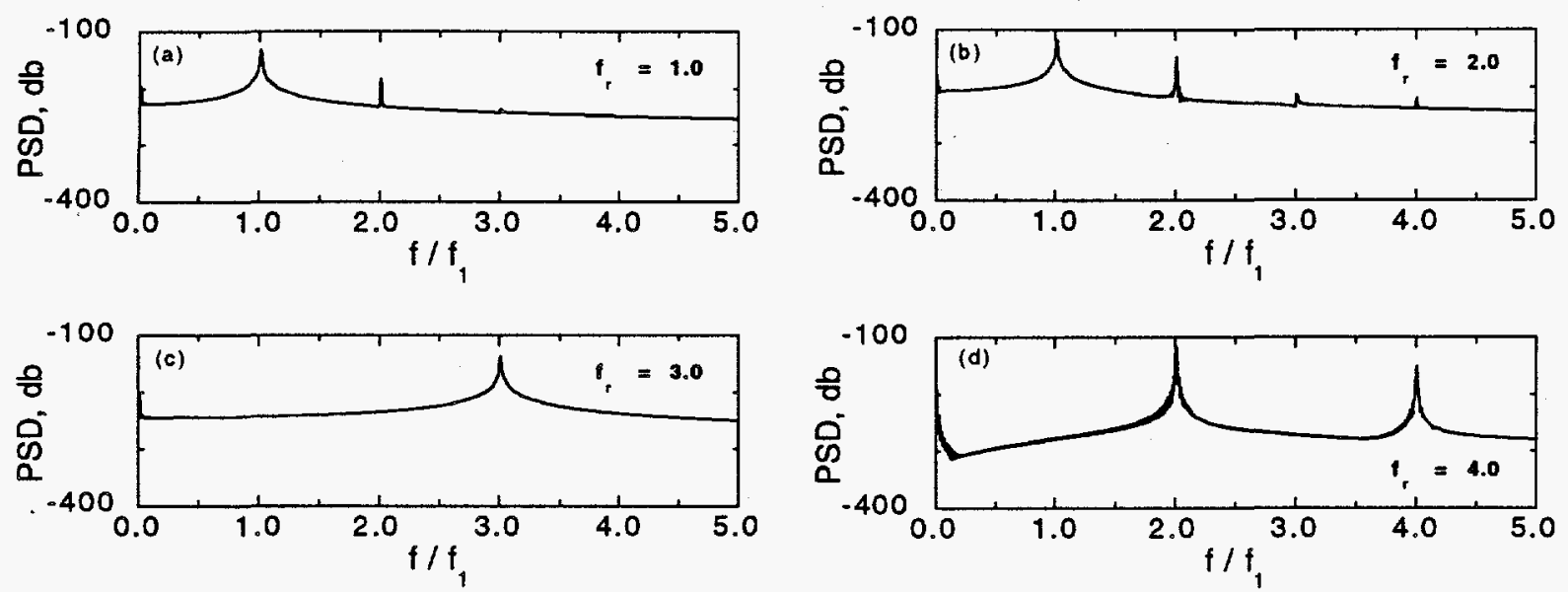

Fig. 12. Power spectral densities of cable displacement at $x=0.125$ with excitation amplitude $w_{0}=0.0003$ and other parameters the same as in Fig. 10

Figure 12 shows power spectral densities of cable displacement at $\mathrm{x}=0.125$ and excitation amplitude $\mathrm{w}_{0}=0.0003$, and illustrates that for $\mathrm{f}_{\mathrm{r}}=1.0$ and 3.0 , there are harmonic peaks due to external resonances (Figs. 12a and 12c); whereas for $f_{r}=2.0$ and 4.0 , besides external resonance harmonics, there are parametric resonances at frequency ratio $f / f_{1}=1.0$ for the case of $f_{r}=2.0$ (Fig. 12b), and at frequency ratio $f / f_{1}=2.0$ for the case of $f_{r}=4.0$ (Fig. $12 \mathrm{~d}$ ).

To better understand the effects of excitation amplitude $\mathrm{w}_{0}$ on parametric resonances, Fig. 13 gives the RMS displacements of the cable near the excitation frequency ratio $f_{r}=2.0$ within a narrow range of excitation amplitude $w_{0}$, which then displays how the influence of parametric resonance increases with excitation amplitude $\mathrm{w}_{0}$. Corresponding to Fig. 13, time histories of the cable displacement at $\mathrm{x}=0.500$ and $\mathrm{x}=0.125$ are shown in Fig. 14, where amplitudes and frequencies of cable oscillation change as excitation amplitude $\mathrm{w}_{0}$ increases; therefore, effects of parametric resonances become more important to cable motion.

The contribution of each mode to external and parametric resonances with various excitation amplitudes $\mathrm{w}_{0}$ should also be examined. Figure 15 shows time histories of the first and second modes with various excitation amplitudes $\mathrm{w}_{0}$ at $f_{r}=2.0$; the dashed line gives the time histories of the external excitation term $\mathrm{A}_{1}+\mathrm{B}_{\mathrm{r}}$ of the right-hand side of Eq. 35. In Fig. 15a, for excitation amplitude $\mathrm{w}_{0}=$ 0.00018 , both the first and second mode are excited by the external excitation $\left(\omega_{\mathrm{s}}=\right.$ $\omega_{2}$ ), and the amplitude of $q_{1}$ is much lower than that of $q_{2}$. With the increase of excitation amplitude $w_{0}$ to 0.0002 (Fig. 15b), the first mode contains two frequencies $\left(\omega_{1}\right.$ and $\left.\omega_{2}\right)$, and is affected by both parametric and external 


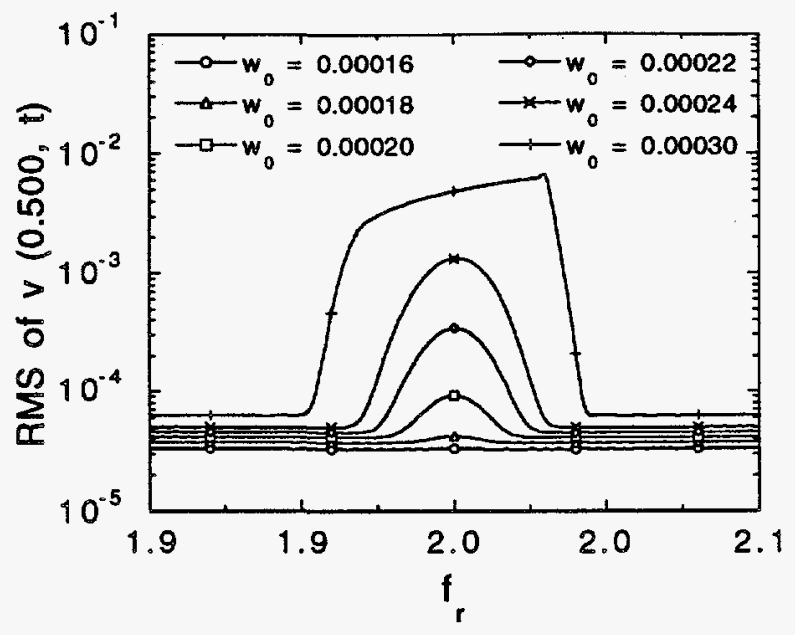

Fig. 13. RMS displacements of cable at $x=$ 0.500 near excitation frequency ratio $f_{r}=2.0$, with various excitation amplitudes $w_{0}(\alpha=$ $0.2 \pi, \beta=0.02, \zeta=0.02, \mu=500$ )
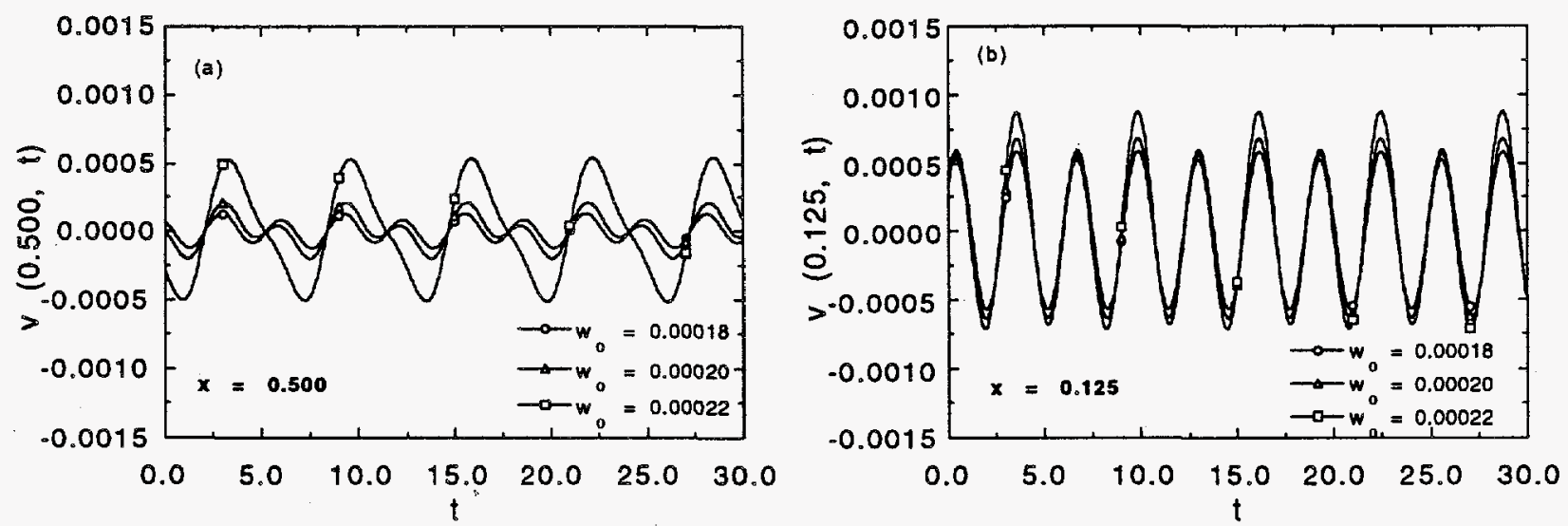

Fig. 14. Time histories of cable displacements with a fixed frequency ratio $f_{r}=2.0$ and various excitation amplitudes $w_{0}$ (other parameters are the same as in Fig. 10)

excitations. If the excitation amplitude is increased to $\mathrm{w}_{0}=0.00022$ (Fig. 15c), the first mode seems to have a single frequency $\omega_{1}$ and parametric resonance dominates the first-mode oscillation. The amplitude of $\mathrm{q}_{1}$ increases but is still lower than that of $q_{2}$. When the excitation amplitude reaches $\mathrm{w}_{0}=0.0003$ (Fig. $15 \mathrm{~d}$ ), not only one frequency appears in the first-mode oscillation, but also its amplitude becomes much greater than that of the second mode. In this case, the first-mode oscillation, which reflects parametric resonance, will dominate cable motion and the effects of external resonance become negligible. 

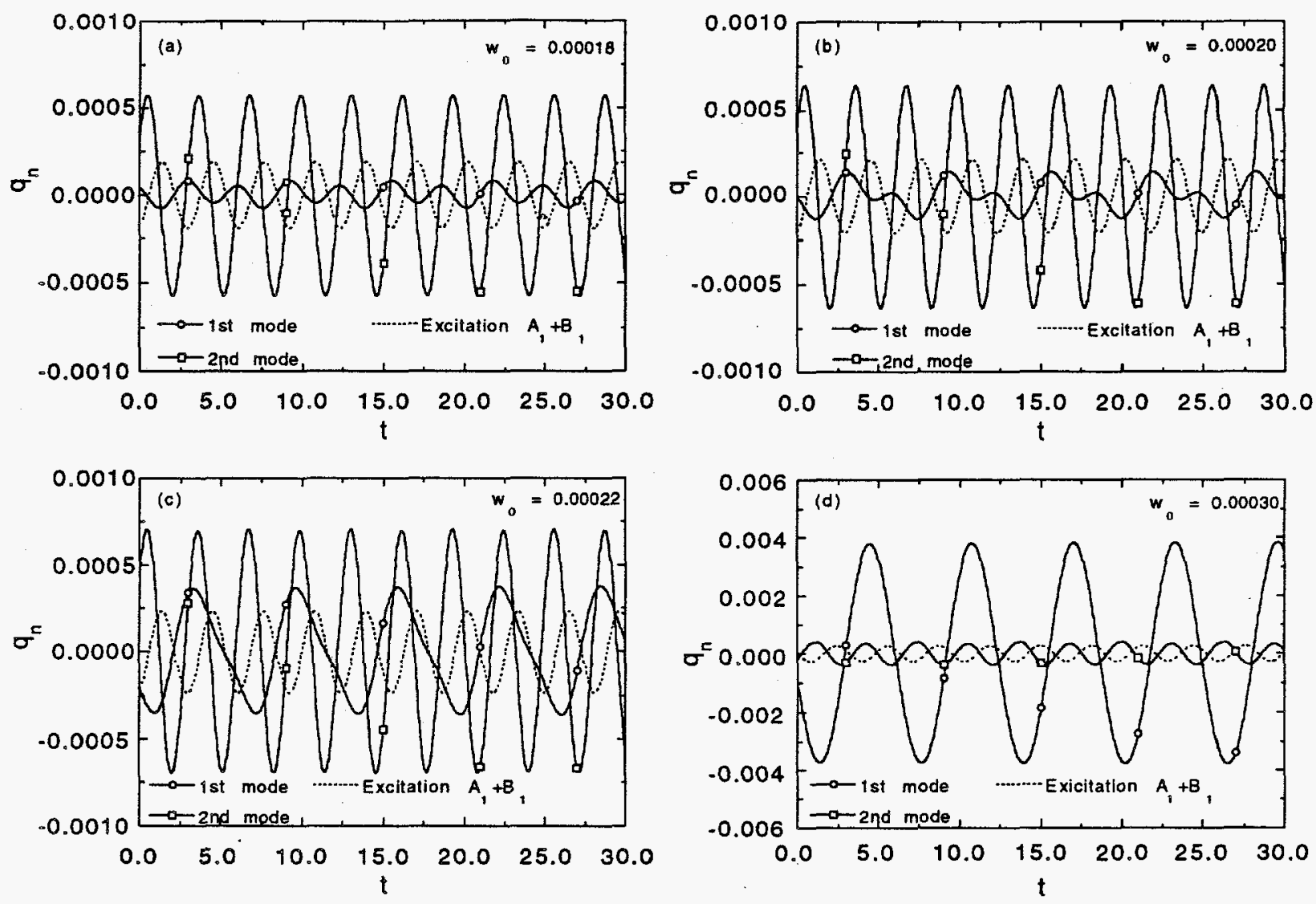

Fig. 15. Time histories of first two modes with fixed frequency ratio $f_{r}=2.0$ and various excitation amplitudes (other parameters are the same as in Fig. 10)

Figure 16 shows the RMS displacements of the cable near the excitation frequency ratio $f_{r}=2.0$ with a fixed excitation amplitude $w_{0}=0.0003$, but varying tilt angle $\alpha$. From Fig. 16, we note that the effects of parametric resonance increase as $\alpha$ decreases; whereas the effects of external resonance decrease as $\alpha$ decreases. This result is evident from Eqs. 14, 15, and 37. If $\alpha$ is large, excitation parameter e will be less than its critical value and parametric instabilities will never occur. On the other hand, if $\alpha$ is small, excitation parameter e can be very large, the effects of parametric resonance increase and that of the external excitation will decrease. When $\alpha$ is equal to zero, there will be no external excitation. 


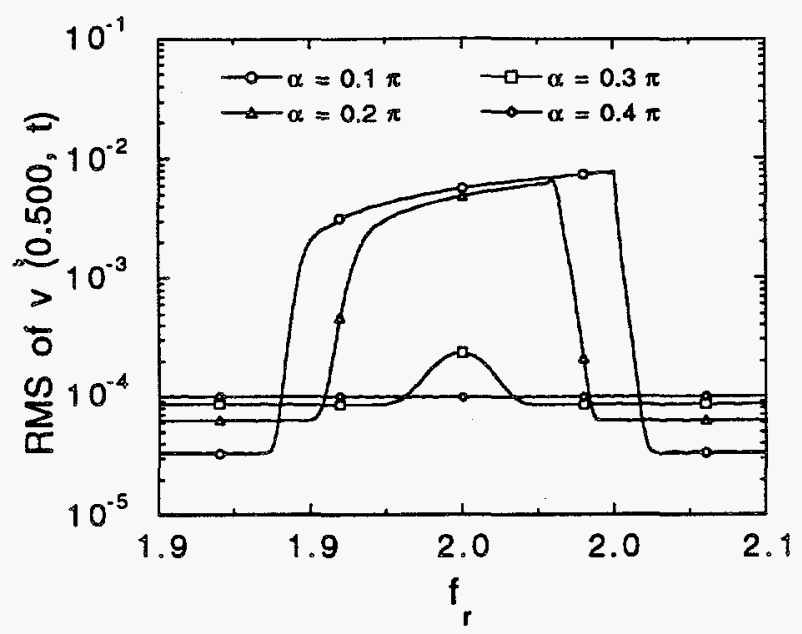

Fig. 16. RMS displacements of cable at $x=$ 0.500 near excitation frequency ratio $f_{r}=2.0$, with various tilt angles $\alpha\left(w_{0}=0.0003, \beta=0.02, \zeta=\right.$ $0.02, \mu=500$ )

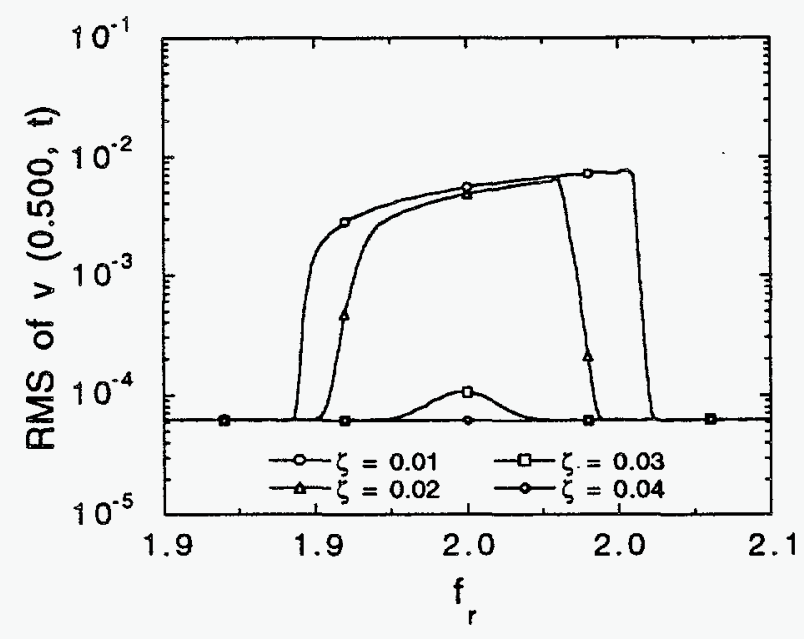

Fig. 17. RMS displacements of cable at $x=$ 0.500 near excitation frequency ratio $f_{r}=2.0$, with various damping ratios $\zeta\left(w_{0}=0.0003, \alpha=\right.$ $0.2 \pi, \beta=0.02, \mu=500$ )

Similar results can be observed in Fig. 17, with fixed $\mathrm{w}_{0}=0.0003$ and $\alpha=0.2 \pi$ and varying damping ratio $\zeta$. The effects of parametric resonance increase with decreasing $\zeta$, because the critical value of excitation parameter e increases with increasing $\zeta$. From Fig. 9 it is evident that damping diminishes the extent of the parametric instability region. 
Many calculations were carried out to investigate the coupling effects of nonlinearities in the cable motion. It was found that, in the presence of external and parametric resonances, the cable was forced to vibrate with a large amplitude, which rendered the influence of modal interactions negligible. However, the nonlinearities of the cable do play an important role in suppressing oscillation amplitude of the cable in the region of parametric instability so that the oscillation amplitude will not progress indefinitely. It is also noted that cubic nonlinearity is more effective in suppressing the oscillation than quadratic nonlinearity. Therefore, the expression of nonlinearity in Eq. 36 is sufficient to simulate the dynamic responses of the elastic cable under external and parametric resonances (Cai and Chen 1994).

In the original stack/wire system, the dominant frequency of the stack oscillation was the third mode (Chen and Cai 1992). It was approximately twice the natural frequency of the wires when wire tensions were within certain ranges. This made parametric resonance possible. Corresponding to the third mode of the stack, the bending displacement amplitudes are very small at the two upper levels (Fig. 1). At the two lower levels, the displacement amplitudes reach $=25-50 \mathrm{~mm}$, which corresponds to a nondimensional excitation amplitude of $\mathrm{w}_{0} \approx$ 0.0004-0.0008. From Fig. 10, at $\mathrm{f}_{\mathrm{r}}=2.0$, parametric resonance will definitely dominate cable motion with those excitation amplitude values. Moreover, tilt angles of the wires for the two lower levels are $\approx 0.1 \pi$ and $0.2 \pi$. From Fig. 16, it is evident that a small tilt angle is more likely to cause parametric resonance. From the factors of excitation amplitude due to stack bending and the tilt angles of the wires, it should not be a surprise that some of the wires, only at the lower two levels, were damaged by large-amplitude oscillations (Chen and Cai 1992, Cai and Chen 1994).

\section{Dynamics of a Stack/Cable System}

\subsection{Coupled Model of a Stack/Cable System}

With the mathematical developments in the previous sections, we can easily derive a coupled nonlinear dynamic model of the wind-induced vibration of a stack (based on an unsteady-flow theory) and the heavy elastic suspended cable whose upper end is subject to bending vibration of the stack.

Consider the original system in Fig. 1. The stack model can be rewritten as

$$
\frac{d^{2} a_{n}}{d t^{2}}+2 \zeta \omega \frac{d a_{n}}{d t}+\omega^{2} a_{n}=\frac{C_{n}}{2(1+\gamma \alpha)}\left(\frac{\rho U^{2} C_{L}^{\prime} D}{m}\right) \cos \left(\omega_{s} t\right),
$$


where

$$
w(z, t)=\sum_{n=1}^{\infty} D a_{n}(t) \psi_{n}(z)
$$

Thus, the cable motion components at the support at the upper end in both axial and transverse directions of the cable $u$ and $v$ are

$$
\mathrm{u}\left(\ell_{\mathrm{k}}, \mathrm{t}\right)=-\mathrm{w}\left(\mathrm{z}_{\mathrm{k}}, \mathrm{t}\right) \cos \alpha
$$

and

$$
\mathrm{v}\left(\ell_{\mathrm{k}}, \mathrm{t}\right)=\mathrm{w}\left(\mathrm{z}_{\mathrm{k}}, \mathrm{t}\right) \sin \alpha
$$

If we let

$$
v(x, t)=v_{1}+v_{2}=\sum_{n=1}^{\infty} q_{n}(t) \phi_{n}(x)+x w
$$

then ordinary differential equations of elastic cables are obtained as

$$
\begin{aligned}
\ddot{q}_{n}+ & 2 \zeta_{n}\left(\frac{\omega_{n}}{\omega}\right) \dot{q}_{n}+\left(\frac{\omega_{n}}{\omega}\right)^{2}\left(\left\{1+\mu\left[w \cos \alpha+I_{0} w \sin \alpha+\frac{1}{2}(w \sin \alpha)^{2}\right]\right\} q_{n}\right. \\
& -\frac{\mu}{n^{2} \pi^{2}}\left(I_{1 n} w \sin \alpha+I_{5 n}\right)\left(I_{2 n} w \sin \alpha+I_{7 n}\right) \sum_{n=1}^{\infty} q_{n} \\
& \left.+\mu\left[I_{6 n}-\frac{1}{n \pi}\left(I_{2 n} w \sin \alpha+I_{7 n}\right)\right] q_{n} \sum_{n=1}^{\infty} q_{n}+\mu I_{3 n} q_{n} \sum_{n=1}^{\infty} q_{n}^{2}\right) \\
= & -I_{4 n} \sin \alpha\left[\ddot{w}+2 \zeta_{n}\left(\frac{\omega_{n}}{\omega}\right) \dot{w}\right] \quad n=1,2,3, \ldots,
\end{aligned}
$$

where $I_{0}, I_{j n}(j=1,2,3, \ldots 7)$ are defined in the Appendix.

\subsection{Dynamic Response of a Stack/Cable System}

The dynamic response of cables can be numerically calculated from Eqs. 51 and 53 as a function of wind speed. Figure 18 gives nondimensional RMS displacements of the cable at $x / \ell_{k}=0.500$ for the first four modes of the stack as a 

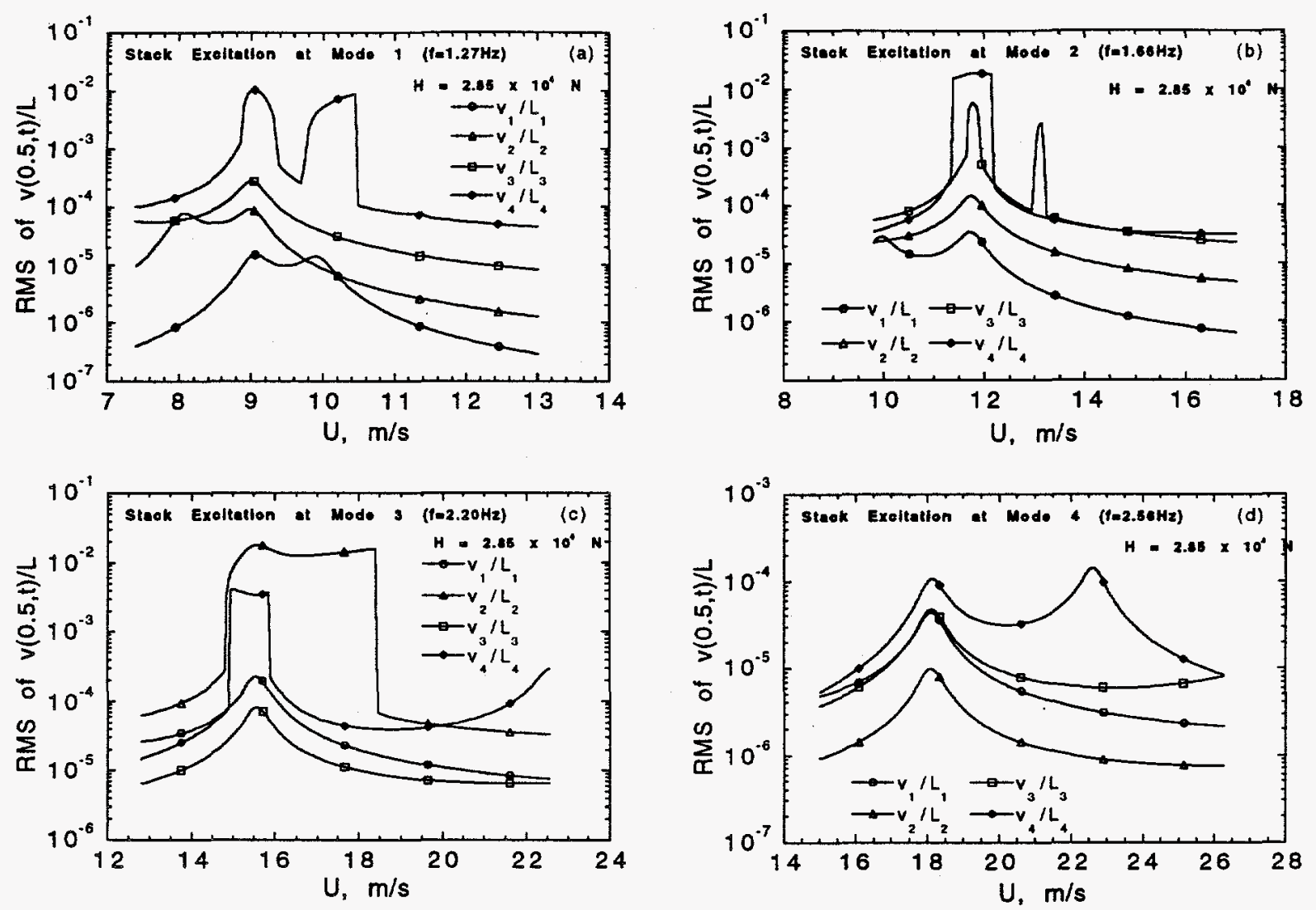

Fig. 18. Nondimensional RMS displacements of cable at $x / \ell=0.5$

function of wind speed $U$, with $S=0.175, \mathrm{C}_{\mathrm{L}}^{\prime}=0.5, \mathrm{H}=2.85 \times 10^{4} \mathrm{~N}, \zeta=0.02$. Regardless of the values of the excitation amplitudes, the cable was subjected to external resonance at lock-in resonances of the stack. When the vortex-shedding frequency is the cable's natural frequency, parametric resonance exists corresponding to the first primary parametric instability frequencies, i.e., primary parametric instabilities occur near $\omega_{\mathrm{s}}=2 \omega_{1}$. These phenomena can be viewed clearly in Fig. 18. In Fig. 18a, which corresponds to mode 1 of the stack $(f=1.27 \mathrm{~Hz}$ ), the first primary parametric resonance occurs in the level-four cable, whose natural frequency is $0.73 \mathrm{~Hz}$ at $U=11 \mathrm{~m} / \mathrm{s}$. In Fig. 18b, which corresponds to mode 2 of the stack $(f=1.66 \mathrm{~Hz}$ ), the first primary parametric resonances occur in the level-four cable, whose natural frequency is $0.73 \mathrm{~Hz}$ at $\mathrm{U}=11 \mathrm{~m} / \mathrm{s}$, and in the level-three cable, whose natural frequency is $0.90 \mathrm{~Hz}$ at $\mathrm{U}=$ $13.5 \mathrm{~m} / \mathrm{s}$. In Fig. 18c, which corresponds to mode 3 of the stack $(f=2.20 \mathrm{~Hz}$ ), the second primary parametric resonances occur in the level-four cable, whose natural frequency is $0.73 \mathrm{~Hz}$ at $U=11 \mathrm{~m} / \mathrm{s}$, and the first primary parametric resonances occur in the level-two cable, whose natural frequency is $1.1 \mathrm{~Hz}$ at $\mathrm{U}=$ $14.5-18.5 \mathrm{~m} / \mathrm{s}$. In Fig. $18 \mathrm{~d}$, which corresponds to mode 4 of the stack $(\mathrm{f}=2.56 \mathrm{~Hz}$ ), no parametric resonances occur under selected stack and cable parameters over the entire range of wind speed. 
Figure 19 shows power spectral densities of cable displacement at $\mathrm{x} / \ell=0.5$, corresponding to Fig. 18.

In Figs. 19a and $19 \mathrm{~b}$, corresponding to the first stack mode $(1.27 \mathrm{~Hz})$ (Fig. 18a), with vortex-shedding frequencies $=1.272$ and $1.399 \mathrm{~Hz}$, all four cable levels show external resonance at lock-in resonance frequency $(1.27 \mathrm{~Hz})$, while the fourth-level cable exhibits parametric resonance with its natural frequency of $\approx 0.7 \mathrm{~Hz}$. In Figs. $19 \mathrm{c}$ and $19 \mathrm{~d}$, corresponding to the second stack mode $(1.66 \mathrm{~Hz})$ (Fig. 18b), besides external resonances for all four-level cables, parametric resonance occurs to the third-level-cable with its natural frequency $\approx 0.9 \mathrm{~Hz}$ (see Fig. $19 \mathrm{~d}, \mathrm{f}_{\mathrm{s}}=1.86 \mathrm{~Hz}$ ). In Figs. $19 \mathrm{e}$ and $19 \mathrm{f}$, corresponding to the third stack mode $(2.2 \mathrm{~Hz}$ ) (Fig. 18c), the vortex-shedding frequency is $\approx 2.2-2.4 \mathrm{~Hz}$; parametric resonances occur to the second-level cable with its natural frequency $\approx 1.1 \mathrm{~Hz}$.

To better understand the effects of cable tension and damping on parametric resonances, RMS displacements of the second-level cable with stack excitation in Mode 3 were calculated for $\zeta=0.02$, and $\mathrm{H}=1.85 \times 10^{4} \mathrm{~N}, 2.85 \times 10^{4} \mathrm{~N}, 4.25 \times 10^{4} \mathrm{~N}$, and $6.50 \times 10^{4} \mathrm{~N}$, where the parametric resonance effect is reduced as the cable tension increases (Fig. 20); and for $\mathrm{H}=2.85 \times 10^{4} \mathrm{~N}$, and $\zeta=0.01,0.02,0.03$, and 0.04 , where the parametric resonance windows subject to wind speed narrowed as cable damping increased (Fig. 21).

To investigate the effects of a fluctuating lift coefficient on parametric resonance, RMS displacements of all four cable levels with stack excitation in Mode 4 were calculated with $\zeta=0.01, \mathrm{H}=2.85 \times 10^{4} \mathrm{~N}$, and $\mathrm{C}_{\mathrm{L}}^{\prime}=1.0$. At the firstlevel cable, very strong parametric primary resonance is demonstrated (see Fig. 22); however, in comparison, Fig. 18d shows no parametric resonance with $\zeta=0.02$ and $C_{L}^{\prime}=0.5$. Therefore, the parametric resonance effects will increase as the fluctuating lift coefficient or excitation parameter increases, a result that has been verified in Fig. 9.

In the original stack/wire system, the dominant frequency of the stack oscillation was the third mode (Chen and Cai 1992). It was approximately twice the natural frequency of the wires when wire tensions were within certain ranges; this made parametric resonance possible. Corresponding to the third mode of the stack, the bending displacement amplitudes are very small at the two upper levels (Fig. 1). At the two lower levels, the displacement amplitudes reach $\approx 25-50 \mathrm{~mm}$. From Fig. $18 \mathrm{c}$, at $\mathrm{U}=14.5-18.5 \mathrm{~m} / \mathrm{s}$, parametric resonance will definitely dominate cable motion. Moreover, it is evident that small dampings and certain tension values are more likely to cause parametric resonance. From these calculation results, it is not surprising that some of the wires, only at the lower two levels, were damaged by large-amplitude oscillations. 

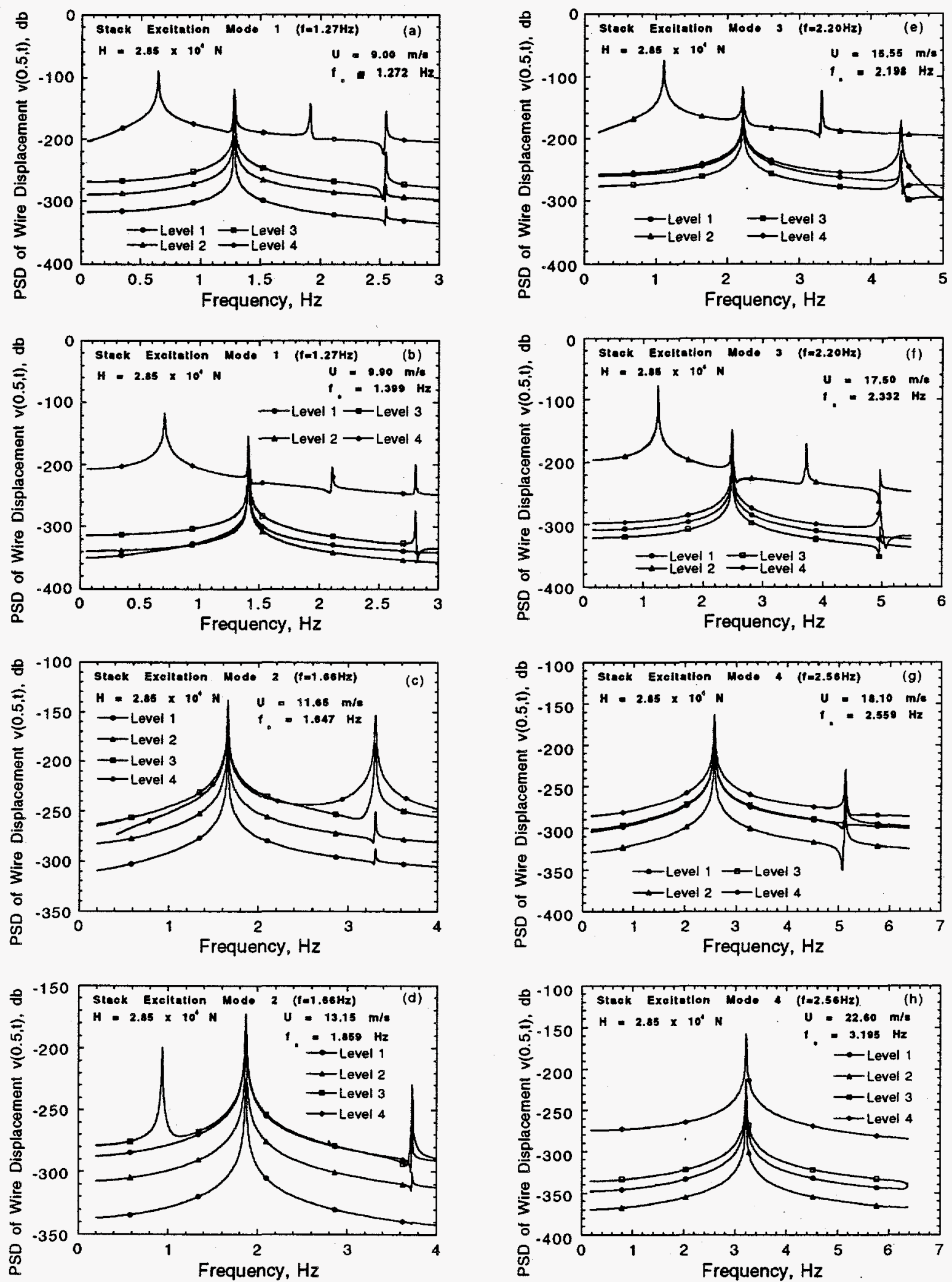

Fig. 19. Power spectral densities of cable displacements at $x / \ell=0.5$ 


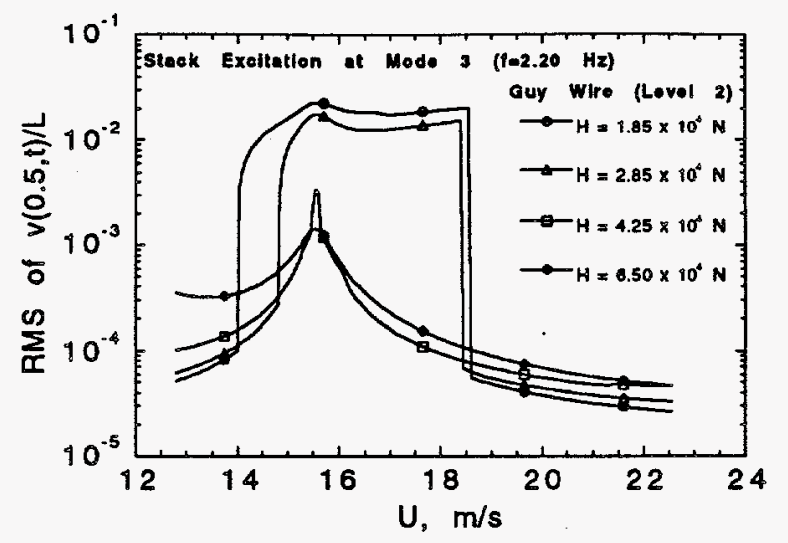

Fig. 20. Nondimensional RMS displacements of cable at $x / \ell=0.5$ with various cable tensions

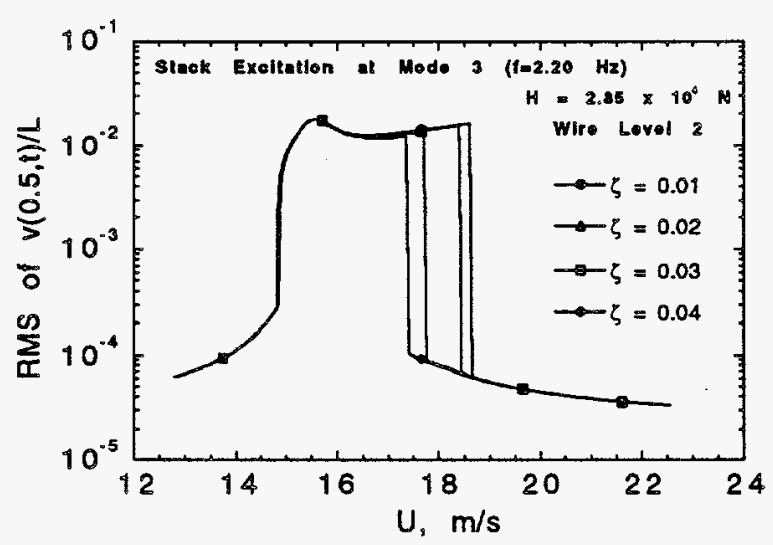

Fig. 21. Nondimensional RMS displacements of cable at $x / \ell=0.5$ with various cable dampings

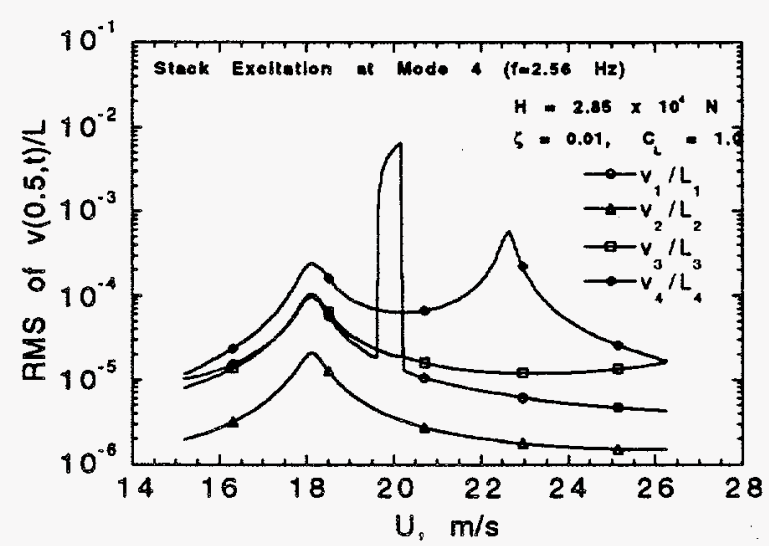

Fig. 22. Nondimensional RMS displacements of cable at $x / \ell=0.5$ with $C_{L}^{\prime}=1.0$ 


\section{Techniques to Suppress Excessive Vibration}

Several methods may be used to reduce vibration amplitude of a stack/wire system to an acceptable level.

(1) Modify flow field. Install spoilers on the lower portion of the stack to prevent the formation of coherent vortex shedding. Once vortex shedding is suppressed at the lower portion of the stack, lock-in resonance will not occur and consequently parametric resonance of the guy wires will also not occur.

(2) Modify structure/wire system. Change structural characteristics, including natural frequencies and mode shapes. This can be accomplished by adding guy wires or other types of supports. The goal is to avoid lock-in resonance.

(3) Add dampers. Various types of dampers can be used to suppress excessive vibration and instability. In particular, dampers can be attached to guy wires to reduce vibration amplitudes.

(4) Combine the above methods. Any combination of the methods can be used.

The logical choice is a modification that is the most economical and effective technique to reduce the amplitude to a level such that the design life is not affected by wind-induced vibration.

\subsection{Short-Term Solution}

The guy wires at the lower two levels can be adjusted so that the tension is approximately one-half of the original values. This will reduce the natural frequencies of the wires to $\approx 70 \%$ of the original values. This modification will reduce the vibration amplitude significantly. Note that lock-in resonance of the stack still exists. However, parametric resonance of the wires may be eliminated. Even if the parametric resonance is not completely eliminated, the vibration amplitudes of the wires are expected to be much smaller.

If adjustment of the wire tension is not completely effective, damping ropes can be installed for the wires at the two lower levels. The damping ropes will further reduce wire vibration. 


\subsection{Long-Term Solution}

Based on this study, a new design to eliminate vortex excitation and parametric resonance can be developed. This solution can be summarized as follows:

- Extend the spoiler to ground level.

- Set the tensions of the guy wires from the bottom level to the top at $3200,4100,4545$, and $5000 \mathrm{~kg}$.

Another step that can be considered is the use of larger-diameter wires. This will increase the natural frequencies of the coupled stack/wire system and increase the strength of the wires. For example, in Model 6, when the crosssectional area of the guy wires is doubled, the lower natural frequencies increase. If the larger wires are used, the tension of the wires from the bottom level to the top can be set to $3200,4100,4545$, and $5000 \mathrm{~kg}$.

The main excitation mechanism is vortex shedding of the stack. When the spoiler is extended to ground level, vortex excitation of the stack is suppressed and vortex-excited lock-in oscillations are not possible. Once vortex shedding is suppressed, stack oscillation will be small. When the stack response amplitude is small, the excitation parameter is also small. In this case, instability regions for parametric resonance will be small (see Fig. 9). Therefore, the probability of having parametric resonance after modification is very small. The main objectives of the modification are to eliminate the excitation of the stack and avoid instability regions. Based on the recommended location and tension of the guy wires, all primary instability regions for parametric resonance can be avoided.

The other excitation is turbulence, which will increase in the presence of a spoiler along the entire stack. However, the response due to turbulence excitation is expected to be much smaller than vortex-excited resonance.

A new stack, modified on the basis of the results of this study, has been installed. The stack is not susceptible to any unacceptable oscillations.

\section{Conclusions}

A stack supported by guy wires at four levels experienced large-amplitude oscillations when wind speed was $>15 \mathrm{~m} / \mathrm{s}$. The response amplitudes depended on the tension of the guy wires. The purpose of this study was to identify the 
excitation mechanisms and develop appropriate modifications to eliminate excessive vibration.

The excitation mechanisms were identified from scoping calculations, analytical prediction with a finite-element code, and observation of the stack/wire response. The natural frequency of the most critical mode of the stack was predicted to be $\approx 2.2 \mathrm{~Hz}$, which is in agreement with observation of the oscillations of the stack and guy wires. The fundamental frequencies of the guy wires at the lower two levels were calculated to be $\approx 1.2 \mathrm{~Hz}$, which agrees well with visual observation. The vortex-shedding frequency at a wind speed of $15 \mathrm{~m} / \mathrm{s}$ was $\approx 2.2-4.4 \mathrm{~Hz}$. Therefore, lock-in resonance associated with the most critical mode of the stack was possible. The fundamental frequencies of the guy wires at the lower two levels were approximately one-half that of the most critical mode of the stack.

The unsteady-flow theory was employed for modeling a fluid/structure system with vortex-shedding-induced vibration of a stack. Motion-dependent fluidforce coefficients were obtained from our previous experimental data. Numerical calculation agreed well with the observation that the stack vibration was excited by vortex shedding at the lower portion associated with the third mode of the stack.

A nonlinear dynamic model was developed for an elastic cable under parametric and external resonances. Numerical analysis was conducted to investigate the dynamic responses of the cable under parametric and external resonance, because the classical analytical approach could not provide an ideal resolution for the system with simultaneous parametric excitation and nonlinear terms. Numerical simulations predicted the existence of parametric and external resonances and their coupling effects on this cable system. In addition, parametric analyses were pursued to understand the dynamic influences of excitation amplitude and frequency, tilt angles of the cable, and system damping on cable motion under parametric and external resonances; the instability regions depend on those parameters and nonlinearities of the system. It was found that excitation amplitudes and tilt angles play very important roles in parametric and external resonances.

A coupled model of wind-induced vibration of a stack, based on an unsteadyflow theory and nonlinear dynamics of heavy elastic suspended cables, was developed in this study. Numerical analysis of the coupled system results in good agreement with observations of the original stack/wire response. The excitation mechanisms of the fluid/structure system were identified as (a) lock-in resonance of the stack by vortex shedding and (b) parametric resonance of the suspended cables by stack motion at the ends of the cable supports. Wind speed, the 
fluctuating lift coefficient, cable tension, and damping are key to parametric resonance of the cables. Adjusting cable tension to certain values, which will change the natural frequency of the cables, may eliminate parametric resonance of the cables. Even if resonance is not completely eliminated, however, the vibration amplitudes of the wires are expected to be much smaller. Installation of damping ropes on the wires will further reduce wire vibration.

\section{Acknowledgments}

This work was sponsored, in part, by the U.S. Department of Energy, Office of Basic Energy Sciences, Division of Engineering and Geosciences, under Contract W-31-109-Eng-38.

\section{References}

Al-Noury, S. I. and Ali, S. A. (1985). Large-amplitude vibrations of parabolic cables. J. Sound Vib. 101(4), 451-462.

Bearman, P. W. (1984). Vortex shedding from oscillating bluff bodies, Annual Rev. Fluid Mech. 16, 195-222.

Benedettini, F. and Rega, G. (1987). Non-linear dynamics of an elastic cable under planar excitation. Int. J. Non-Linear Mech. 22(6), 497-509.

Birkhoff, G. (1953). Formation of vortex streets. J. Appl. Phys. 24, 209-231.

Blevins, R. D. (1979). Formulas for natural frequency and mode shape. Van Nostrand Reinhold Company, New York.

Cai, Y. and Chen, S. S. (1994). Dynamics of elastic cable under parametric and external resonances. J. Eng. Mech. ASCE 120(8), 1786-1802.

Chen, S. S. (1987). Flow-induced vibration of circular cylindrical structures. Hemisphere Publishing Co., New York.

Chen, S. S. and Cai, Y. (1992). Wind induced vibration of a stack. ASME Publication PVP-Vol. 245, pp. 159-170.

Chen, S. S., Zhu, S. and Cai, Y. (1995). An unsteady flow theory for vortex-induced vibration. J. Sound Vib. 184(1), 73-92. 
Chen, S. S., Zhu, S., and Jendrzejczyk, J. A. (1993). Motion-dependent fluid forces acting on tube arrays in crossflow. Argonne National Laboratory Report ANL-93/15.

Dowell, E. H. (1981). Non-linear oscillator models in bluff body aeroelasticity. J. Sound Vib. 75(2), 251-264.

Griffin, O. M. (1982). Vortex streets and patterns. Mech. Eng. 104(3), 56-61.

Hartlen, R. K. and Currie, I. G. (1970). Lift oscillator model of vortex-induced vibration. J. Eng. Mech. 96, 577-591.

Ikegawa, M., Kaiho, M. and Kato, C. (1993). FEM/FDM composite incompressible flow analysis .- two-dimensional flow analysis around moving bodies. JSME Int. J. Series B 36(2), 321-327.

King, R. (1977). A review of vortex shedding research and its application. Ocean Eng. 4, 141-171.

Luongo, A., Rega, G. and Vestroni, F. (1984). Planar non-linear free vibrations of an elastic cable. Int. J. Non-Linear Mech. 19(1), 39-52.

Morkovin, M. V. (1964). Flow around circular cylinders. a kaleidoscope of challenging fluid phenomena. ASME Symp. on Fully Separated Flows, Philadelphia, PA, ASME, New York, pp. 102-118.

Narasimha, R. (1968). Non-linear vibration of an elastic string. J. Sound Vib. 8(1), 134-146.

Perkins, N. C. (1992). Modal interactions in the non-linear response of elastic cables under parametric/external excitation. Int. J. Non-Linear Mech. 27(2), 233-250.

Rao, G. V. and Iyengar, R. N. (1991). Internal resonance and non-linear response of a cable under periodic excitation. J. Sound Vib. 149(1), 25-41.

Rega, G., Vestroni, F. and Benedettini, F. (1984). Parametric analysis of large amplitude free vibrations of a suspended cable. Int. J. Non-Linear Mech. 20(2), 95-105.

Sadaoka, N. and Umegaki, K. (1993). A numerical method to calculate flow: induced vibrations in a turbulent flow. ASME Publication PVP-Vol. 258, 83-94. 
Sarpkaya, T. (1979). Vortex-induced oscillations - a selective review. J. Appl. Mech. 46, 241-258.

Sarpkaya, T. and Isaacson, M. (1981). Mechanics of wave forces on offshore structures. Van Nostrand Reinhold Company, New York.

Scanlan, R. H. (1977). The action of flexible bridges under wind, 1: flutter theory. J. Sound Vib. 60(2), 187-199.

Simiu, E. and Scanlan, R. H. (1986). Wind effects on structures. John Wiley \& Sons, New York.

Skop, R. A. and Griffin, R. A. (1973). A model for the vortex-excited resonant response of bluff cylinders. J. Sound Vib. 27(2), 225-233.

Staubli, T. (1983). Calculation of the vibration of an elastically mounted cylinder using experimental data from a forced oscillation. ASME J. Fluids Eng. 105, 225229.

Stoker, J. J. (1950). Nonlinear Vibrations. Interscience Publishers, Inc., New York.

Takahashi, K. (1991). Dynamic stability of cables subjected to an axial periodic load. J. Sound Vib. 144(2), 323-330.

Takahashi, K. and Konishi, Y. (1987). Non-linear vibrations of cables in three dimensions, part I: nonlinear free vibrations. J. Sound Vib. 118(1), 69-84.

Vickery, B. J. and Basu, R. L. (1983). Across-wind vibrations of structures of circular cross-section, part 1: development of a model for two-dimensional conditions. J. Wind Eng. Ind. Aerodyn. 12, 49-73. 


\section{Appendix}

Expressions for Dimensionless Coefficients in Eqs. 32 and 35

$$
\begin{aligned}
& I_{0}=\int_{0}^{1} \frac{d y}{d x} d x, \\
& I_{1 n}=\int_{0}^{1} \phi_{n}(x) d x= \begin{cases}\frac{2 \sqrt{2}}{n \pi} & n=1,3,5, \ldots \\
0 & n=2,4,6, \ldots\end{cases} \\
& I_{2 n}=\int_{0}^{1} \phi_{n}^{\prime \prime}(x) d x= \begin{cases}-2 \sqrt{2} n \pi & n=1,3,5, \ldots, \\
0 & n=2,4,6, \ldots,\end{cases} \\
& I_{3 n}=\int_{0}^{1} \frac{1}{2} \phi_{n}^{\prime 2}(x) d x=\frac{n^{2} \pi^{2}}{2} \quad n=1,2,3, \ldots, \\
& I_{4 n}=\int_{0}^{1} x \phi_{n}(x) d x=\frac{-\sqrt{2}}{n \pi} \cos n \pi \quad n=1,2,3, \ldots, \\
& \mathrm{I}_{5 \mathrm{n}}=\int_{0}^{1} \frac{\mathrm{dy}}{\mathrm{dx}} \phi_{\mathrm{n}}(\mathrm{x}) \mathrm{dx} \quad \mathrm{n}=1,2,3, \ldots, \\
& I_{6 n}=\int_{0}^{1} \frac{d y}{d x} \phi_{n}^{\prime}(x) d x \quad n=1,2,3, \ldots, \\
& I_{7 n}=\int_{0}^{1} \frac{d y}{d x} \phi_{n}^{\prime \prime}(x) d x \quad n=1,2,3, \ldots, \\
& \mathrm{C}_{0}=1+\mu\left[\frac{1}{4} \mathrm{v}_{0}^{2}\left(1-\cos 2 \omega_{\mathrm{r}} \mathrm{t}\right)+\left(\mathrm{u}_{0}+\mathrm{I}_{0} \mathrm{v}_{0}\right) \sin \omega_{\mathrm{r}} \mathrm{t}\right], \\
& C_{1 n}=-\frac{\mu}{n^{2} \pi^{2}}\left(I_{1 n} v_{0} \sin \omega_{r} t+I_{5 n}\right)\left(I_{2 n} v_{0} \sin \omega_{r} t+I_{7 n}\right), \\
& C_{2 n}=\mu\left[I_{6 n}-\frac{1}{n \pi}\left(I_{2 n} v_{0} \sin \omega_{r} t+I_{7 n}\right)\right], \\
& C_{3 n}=\mu I_{3 n} \text {, }
\end{aligned}
$$




$$
\begin{aligned}
& A_{n}=\omega_{\mathfrak{r}}^{2} I_{4 n} v_{0}, \\
& B_{n}=-2 \zeta_{n} n \omega_{r} I_{4 n} v_{0},
\end{aligned}
$$$$
\mathrm{n}=1,2,3, \ldots
$$ 


\section{Distribution for ANL-95/30}

Internal

Y. Cai (10)

W. J. Shack

R. D. Carlson

R. A. Valentin

S. S. Chen (10)

M. W. Wambsganss (3)

H. Drucker

R. W. Weeks

C. A. Malefyt

TIS Files

R. B. Poeppel

\section{External}

DOE-OSTI for distribution per UC-406 (45)

ANL Libraries

ANL-E (2)

ANL-W

Manager, Chicago Field Office, DOE

Director, Technology Management Div., DOE-CH

D. L. Bray, DOE-CH

T. Crawford, DOE-CH

Energy Technology Division Review Committee:

H. K. Birnbaum, University of Illinois, Urbana

R. C. Buchanan, University of Cincinnati, Cincinnati, OH

M. S. Dresselhaus, Massachusetts Institute of Technology, Cambridge, MA

B. G. Jones, University of Illinois, Urbana

C.-Y. Li, Cornell University, Ithaca, NY

S.-N. Liu, Fremont, CA

R. E. Smith, Altran Corp., Huntersville, NC

J. S. Coleman, DOE, Washington, DC

O. P. Manley, DOE, Washington, DC

F. C. Moon, Cornell University, Ithaca, NY

J. R. Welty, DOE, Washington, DC 